\title{
Spectroscopy of the Young Stellar Association Price-Whelan 1: Origin in the Magellanic Leading Arm and Constraints on the Milky Way Hot Halo
}

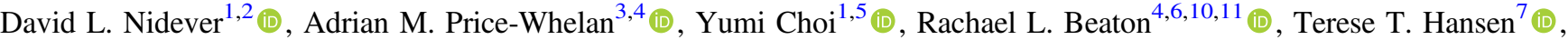 \\ Douglas Boubert $^{8}$, David Aguado ${ }^{8}$ (D), Rana Ezzeddine ${ }^{9}$ (iD), Semyeong Oh $^{8}$ (iD), and N. Wyn Evans ${ }^{8}$ \\ ${ }^{1}$ Department of Physics, Montana State University, P.O. Box 173840, Bozeman, MT 59717-3840, USA; dnidever@montana.edu \\ ${ }^{2}$ National Optical Astronomy Observatory, 950 North Cherry Avenue, Tucson, AZ 85719, USA \\ ${ }^{3}$ Center for Computational Astrophysics, Flatiron Institute, 162 Fifth Avenue, New York, NY 10010, USA \\ ${ }^{4}$ Department of Astrophysical Sciences, Princeton University, 4 Ivy Lane, Princeton, NJ 08544, USA \\ ${ }^{5}$ Space Telescope Science Institute, 3700 San Martin Drive, Baltimore, MD 21218, USA \\ ${ }^{6}$ The Observatories of the Carnegie Institution for Science, 813 Santa Barbara Street, Pasadena, CA 91101, USA \\ ${ }^{7}$ Mitchell Institute for Fundamental Physics and Astronomy and Department of Physics and Astronomy, Texas A\&M University, College Station, \\ TX 77843-4242, USA \\ ${ }^{8}$ Institute of Astronomy, Madingley Road, Cambridge CB3 0HA, UK \\ ${ }^{9}$ Department of Physics and Kavli Institute for Astrophysics and Space Research, Massachusetts Institute of Technology, Cambridge, MA 02139, USA \\ Received 2019 August 10; revised 2019 October 12; accepted 2019 October 29; published 2019 December 16
}

\begin{abstract}
We report spectroscopic measurements of stars in the recently discovered young stellar association Price-Whelan 1 (PW 1), which was found in the vicinity of the Leading Arm (LA) of the Magellanic Stream (MS). We obtained Magellan+MIKE high-resolution spectra of the 28 brightest stars in PW 1 and used The Cannon to determine their stellar parameters. We find that the mean metallicity of PW 1 is $[\mathrm{Fe} / \mathrm{H}]=-1.23$ with a small scatter of 0.06 dex and the mean $\mathrm{RV}$ is $V_{\text {hel }}=276.7 \mathrm{~km} \mathrm{~s}^{-1}$ with a dispersion of $11.0 \mathrm{~km} \mathrm{~s}^{-1}$. Our results are consistent in $T_{\text {eff }}, \log g$, and $[\mathrm{Fe} / \mathrm{H}]$ with the young and metal-poor characteristics $(116 \mathrm{Myr}$ and $[\mathrm{Fe} / \mathrm{H}]=-1.1)$ determined for PW 1 from our discovery paper. We find a strong correlation between the spatial pattern of the PW 1 stars and the LA II gas with an offset of $-10^{\circ} .15$ in $L_{\mathrm{MS}}$ and $+1^{\circ} .55$ in $B_{\mathrm{MS}}$. The similarity in metallicity, velocity, and spatial patterns indicates that PW 1 likely originated in LA II. We find that the spatial and kinematic separation between LA II and PW 1 can be explained by ram pressure from Milky Way (MW) gas. Using orbit integrations that account for the LMC and MW halo and outer disk gas, we constrain the halo gas density at the orbital pericenter of PW 1 to be $\boldsymbol{n}_{\text {halo }}(17 \mathrm{kpc})=2.7_{-2.0}^{+3.4} \times 10^{-3}$ atoms $\mathrm{cm}^{-3}$ and the disk gas density at the midplane at $20 \mathrm{kpc}$ to be $\boldsymbol{n}_{\text {disk }}(20 \mathrm{kpc}, 0)=6.0_{-2.0}^{+1.5} \times 10^{-2}$ atoms $\mathrm{cm}^{-3}$. We, therefore, conclude that PW 1 formed from the LA II of the MS, making it a powerful constraint on the MW-Magellanic interaction.
\end{abstract}

Unified Astronomy Thesaurus concepts: Open star clusters (1160); Halo stars (699); Magellanic Stream (991); High resolution spectroscopy (2096)

Supporting material: data behind figure

\section{Introduction}

The gaseous Magellanic Stream (MS) and its Leading Arm (LA) component, which, respectively, trail and lead the Magellanic Clouds (MCs) in their orbit about the Milky Way (MW), are one of the most prominent HI features in the sky (Wannier et al. 1972; Mathewson et al. 1974; Putman et al. 1998, 2003; Brüns et al. 2005; Nidever et al. 2008; Stanimirović et al. 2008). Together, the MS and LA stretch over $200^{\circ}$ from end to end (Nidever et al. 2010), and are a prototypical example of a gaseous stream stripped from a satellite galaxy in the process of being accreted onto the MW.

The LA is composed of four main complexes (LA I-IV; Brüns et al. 2005; For et al. 2013), is shorter than the trailing stream, and has a more irregular shape, the latter two characteristics being likely due to the effects of ram pressure. In fact, many of the LA cloudlets show head-tail shape (e.g., McClure-Griffiths et al. 2008). Two of the components, LA II and III, lie above the Galactic plane (i.e., at positive Galactic latitudes), suggesting that these complexes have already passed through the Galactic midplane. The formation of the LA has

\footnotetext{
${ }^{10}$ Hubble Fellow.

11 Carnegie-Princeton Fellow.
}

been a topic of great debate, but the proposed formation mechanisms have generally broken down into three primary physical processes: tidal stripping (e.g., Gardiner \& Noguchi 1996; Connors et al. 2006; Besla et al. 2012; Diaz \& Bekki 2012), ram pressure stripping (e.g., Mastropietro et al. 2005), and stellar feedback (e.g., Olano 2004; Nidever et al. 2008). Each formation mechanism has its own strengths and weaknesses in terms of explaining the LA morphology and kinematics. In the end, the formation of the LA is likely a combination of all three of these mechanisms, albeit their relative importance and chronological sequence remains an active topic of research (see the recent review by D'Onghia \& Fox 2016).

With high-precision proper motion measurements of the MCs (e.g., Kallivayalil et al. 2006, 2013; Besla et al. 2007; Helmi et al. 2018), the first-infall scenario, e.g., that the MCs are on their first passage around the MW, has become a widely accepted model to explain both the dynamical evolution of the MCs and their interaction with the MW. In this scenario, the MCs were dynamically bound long before they fell into the MW potential $\sim 1$ Gyr ago (Besla et al. 2012). Many threads of observational evidence suggest that the MCs had the first strong gravitational interaction $\sim 2-3$ Gyr ago (e.g., Harris \& Zaritsky 2004, 2009; Weisz et al. 2013) and then experienced a 
direct collision a few hundred Myr ago (e.g., Olsen et al. 2011; Besla et al. 2012; Noël et al. 2015; Choi et al. 2018a, 2018b; Zivick et al. 2018). The majority of gas in the LA and MS was likely stripped during the former interaction, while the gas in the Magellanic Bridge was likely stripped off during the latter. However, the exact timing, mass, and origin (e.g., LMC or $\mathrm{SMC}$ ) for the gas required to form the LA, MS, and the Magellanic Bridge remain unknown (Pardy et al. 2018).

Due to the differences in the star formation and baryon cycle histories of the LMC and SMC, the chemical abundances of the gas provide critical clues to understanding the origin and evolution of the Magellanic system. Abundances for high-velocity clouds associated with the MS have been measured using absorption along the line of sight (LOS) to a bright background source (a quasar or hot star), but appropriate sight lines are limited in number and location to produce a global characterization. A number of Hubble Space Telescope studies have found that the MS has a mean metallicity of $[\mathrm{Fe} / \mathrm{H}] \approx-1 \mathrm{dex}$ across its length (Fox et al. 2010, 2013b), although there is evidence for a more metal-rich component (Fox et al. 2013b; Richter et al. 2013). The Magellanic Bridge, the gas between the MCs, also has a metallicity of $\approx-1$ (Lehner et al. 2008) as does the LA (Lu et al. 1998; Fox et al. 2018; Richter et al. 2018). The consistency in the metallicities for these distinct gaseous features that lead, trail, and connect the MCs suggests that they share a common originating system: the MCs themselves. The current peak stellar metallicities of the LMC and $\mathrm{SMC}$ are $[\mathrm{Fe} / \mathrm{H}]=-0.6 \mathrm{dex}$ and $[\mathrm{Fe} / \mathrm{H}]=-1.0 \mathrm{dex}$, and even higher in the innermost regions, which have ongoing star formation, reaching $[\mathrm{Fe} / \mathrm{H}]=-0.2$ and $[\mathrm{Fe} / \mathrm{H}]=-0.7$, respectively (Nidever et al. 2019a). The current mean metallicity of the gas in the $\mathrm{LMC}$ and $\mathrm{SMC}$ is also high- $[\mathrm{Fe} / \mathrm{H}]=-0.2$ and $[\mathrm{Fe} / \mathrm{H}]=-0.6$, respectively (Russell \& Dopita 1992)—consistent with the most metal-rich stars. However, the gas components that formed the LA and MS were stripped some time ago and, therefore, their chemistry should be compared to the MCs in the past. According to Pagel \& Tautvaisiene (1998) and Harris \& Zaritsky (2004) the MC metallicities were $\sim 0.3$ dex lower $\sim 2 \mathrm{Gyr}$ ago making the SMC's metallicity consistent with those measured in the gaseous MS, LA, and Bridge today.

While the MCs are known to have extensive stellar peripheries (e.g., Muñoz et al. 2006; Majewski et al. 2009; Nidever et al. 2011, 2019b), no stars have been detected in the MS or LA despite many attempts (e.g., Philip 1976a, 1976b; Recillas-Cruz 1982; Brueck \& Hawkins 1983; Kunkel et al. 1997; Guhathakurta \& Reitzel 1998). Recent star formation in the LA is expected because molecular hydrogen is ubiquitous in the LA (e.g., Richter et al. 2018) and shock compression caused by gas in both the Galactic disk and halo is anticipated. Indeed, Casetti-Dinescu et al. (2014) discovered a number of young stars in the region of the LA that had RVs consistent with being formed in the LA gas, initially confirmed by followup, high-resolution spectroscopy with Magellan+MIKE that enabled chemical abundance measurements of these stars. However, more recent high-resolution spectroscopy and orbital analyses incorporating Gaia DR2 (Brown et al. 2018) proper motions from the same team determined that the stars were incompatible with a Magellanic origin (Zhang et al. 2019).

While the initial claims of LA-associated young stars were invalidated with Gaia DR2 proper motions, Price-Whelan et al. (2019, hereafter Paper I) discovered a young stellar association ("Price-Whelan 1," hereafter PW 1) using Gaia proper motions in the vicinity of the LA. In Paper I, PW 1 was found to be young, metal-poor, and likely disrupting with a sky position similar to that of LA II. By comparing to stellar evolution models, Paper I measured an age of $\sim 116 \mathrm{Myr}$, distance of $\sim 29 \mathrm{kpc}$, metallicity of $[\mathrm{Fe} / \mathrm{H}]=-1.14$, and total present-day stellar mass of $\sim 1200 M_{\odot}$. Although the radial velocity (RV) of the cluster was unknown at the time, the range of possible orbits strongly suggested an association with the MCs and a passage through the outer MW disk $\sim 116$ Myr ago. However, there are many H I structures in the vicinity of PW 1 (Figure 1), each with a unique RV signature. Associating PW 1 with a particular gaseous substructure therefore requires spectroscopic RV measurements of the cluster stars.

In this paper, we analyze the stellar parameters and mean kinematics of the stars in PW 1 to better constrain the properties and origin of this young stellar association. We also use the kinematics of PW 1 in an orbital analysis to constrain the density of the MW hot halo and outer gas disk. The layout of the paper is as follows: Section 2 describes the observations and data reduction. In Section 3, we present the procedures for deriving RVs and stellar parameters. Our main results are detailed in Section 4 and discussed in Section 5. Finally, our main conclusions are summarized in Section 6.

\section{Observations and Reductions}

We obtained spectra for the brightest 28 PW 1 stars and 6 standard stars using the Magellan Inamori Kyocera Echelle (MIKE) spectrograph (Bernstein et al. 2003) on Magellan-Clay at Las Campanas Observatory on 2019 April 25, 26, and 30 and May 1. Some of the stars were also observed on 2018 December 26-28 using the Goodman Spectrograph (Clemens et al. 2004) at the SOAR Telescope with consistent results. Table 1 presents the names, coordinates, magnitudes, signal-to-noise ratio $(\mathrm{S} / \mathrm{N})$, and proper motions for each target. Figure 2 shows their location in a Gaia color-magnitude diagram (red open circles) relative to all PW 1 candidates (blue) and field stars (black). Observations were taken with the 0 " 7 slit and $2 \times 2$ pixel binning, such that the native resolution for each spectrum is $R=31,000$. Exposure times ranged from 70 to $1060 \mathrm{~s}$ under generally good conditions with an average seeing of 0 !"7. At the native MIKE resolution, $\mathrm{S} / \mathrm{N}$ per resolution element (3.15 pixels) for the PW 1 stars is $10-24$ with a median of $\sim 18$, although the $\mathrm{S} / \mathrm{N}$ per pixel is increased by $3.16 \times$ by rebinning the $1 \mathrm{D}$ extracted spectra (see below). Six of the stars have low $\mathrm{S} / \mathrm{N}$ due to moderately cloudy conditions (PW1-00-PW1-05) and are generally excluded from detailed analysis. An additional high-S/N spectrum was obtained for PW1-00 (the brightest PW 1 member) that is suitable for precise chemical abundance work, but this analysis is reserved for future work.

The observations were processed using the MIKE pipeline, ${ }^{12}$ which is part of the CarPy spectroscopic reduction package that uses algorithms described in Kelson et al. (2000) and Kelson (2003). The MIKE pipeline performs image processing (bias removal, flat-fielding) as well as extracting the spectra, determining a wavelength solution, subtracting the sky, and coadding multiple exposures of single objects. The multi-order reduced spectra were then merged into a single spectrum and normalized using the $\operatorname{IRAF}^{13}$ tasks continuum and scombine. While

\footnotetext{
$\overline{12}$ https://code.obs.carnegiescience.edu/mike

13 IRAF is distributed by the National Optical Astronomy Observatories, which are operated by the Association of Universities for Research in Astronomy, Inc., under cooperative agreement with the National Science Foundation.
} 


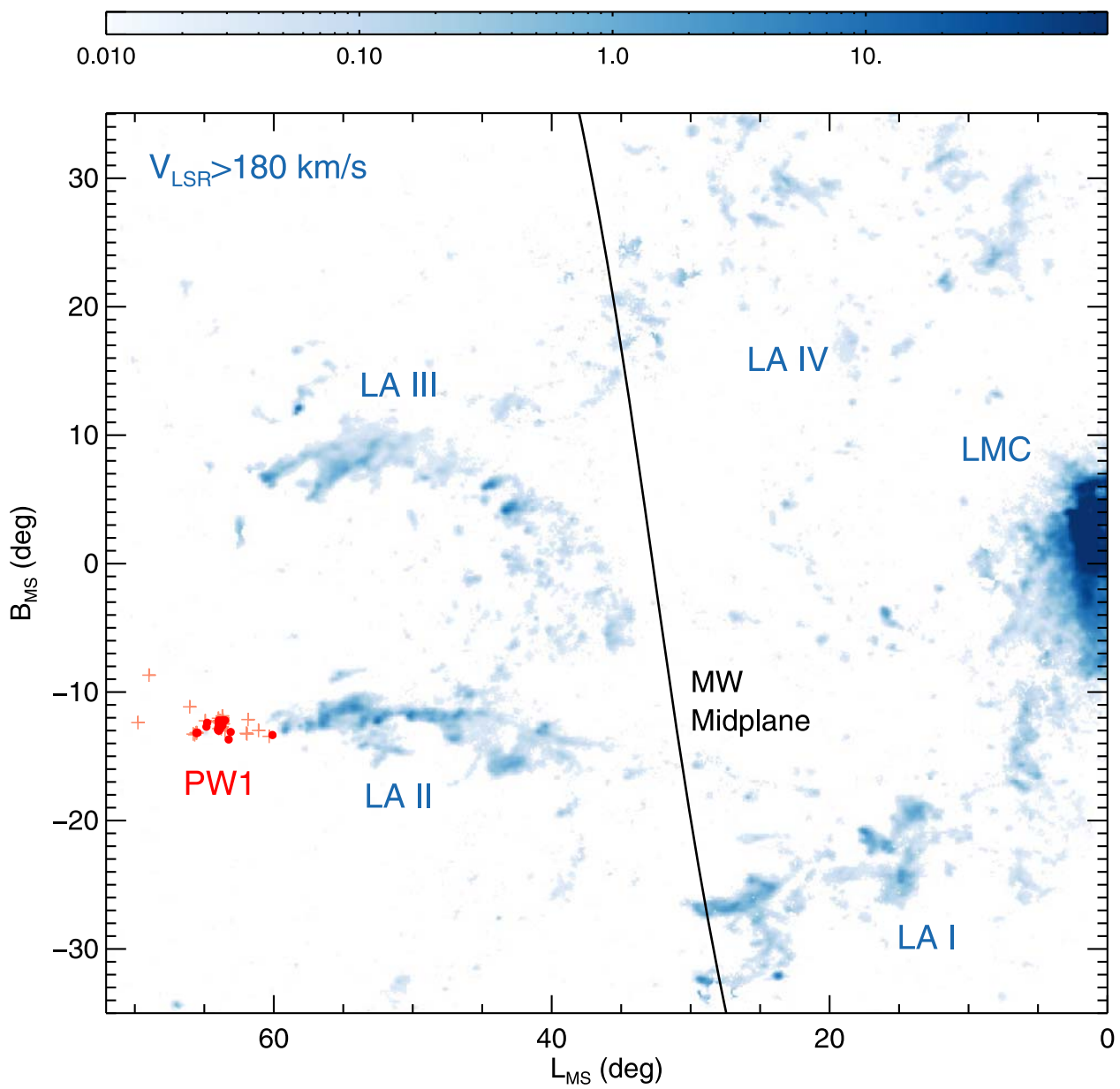

Figure 1. Map of the Leading Arm region. The GASS H I column density (in units of $10^{19}$ atoms $\mathrm{cm}^{-2}$ ) is shown in blue with the LA I-IV and LMC labeled. The 28 PW1 stars for which we obtained MIKE spectra shown as filled red circles while the light-red crosses are 43 additional PW1 candidates based on photometry and Gaia DR2 proper motions. The MW midplane is shown by a solid black line.

MIKE produces spectra from the blue and red arms, only blue spectra with a wavelength range of $3550-5060 \AA$ were used in our analysis. Figure 3 shows example spectra for two PW 1 stars and the standard star HD 146775.

Finally, the full MIKE resolution was significantly higher than needed for our science goals. Therefore, we rebinned the spectra with a bin size of 10 native MIKE pixels which increased the $\mathrm{S} / \mathrm{N}$ per pixel by $\sim 3.16 \times$. This had little impact on the spectral features as they are already quite broad in these hot, B-type stars.

\section{Data Analysis}

\subsection{Radial Velocities}

A RV was determined for each star via a two phase process. First, we determine an initial RV for each source by crosscorrelating the unsmoothed MIKE spectra (excluding the region below $3665 \AA$ ) with a hot synthetic stellar spectrum having stellar parameters $\left(T_{\text {eff }}, \log g,[\mathrm{Fe} / \mathrm{H}]\right)=(15,000 \mathrm{~K}$, $4.0,-1.0)$ and set to the the resolution and logarithmic wavelength scale of the MIKE spectra. Second, the RVs were then redetermined using its best-fit model as the crosscorrelation template after the star-by-star stellar parameters were determined following the procedure described in Section 3.2. The second iteration provided similar, but more precise and accurate RV solutions.
We determine the RV uncertainties using a Monte Carlo scheme. Mock observations were generated for each star by adding Gaussian noise similar to that observed in the science spectrum to its best-fit model spectrum. One hundred mock observations were generated for each star, the RV determined for each with the method described above, and the uncertainty calculated as the robust standard deviation of these values. The typical RV uncertainty from this procedure is $\sim 3 \mathrm{~km} \mathrm{~s}^{-1}$ but the uncertainty increases roughly with the inverse of $\mathrm{S} / \mathrm{N}$.

We compared our RVs for the six bright standard stars to literature values and find a median offset of $-2.0 \mathrm{~km} \mathrm{~s}^{-1}$, which is consistent within the uncertainties of our measurements.

\subsection{Stellar Parameters}

We use The Cannon ${ }^{14}$ (Ness et al. 2015; Casey et al. 2016) to determine stellar parameters $\left(T_{\text {eff }}, \log g,[\mathrm{Fe} / \mathrm{H}]\right)$ for our 28 spectra. The Cannon is a data-driven model for stellar spectra in which the stellar flux (at a given wavelength) is parameterized as a polynomial function of stellar parameters and abundances (i.e., "labels"). The Cannon "model" is trained on a set of spectra (observed or synthetic) with well-known labels (i.e., the "training set") and can then be used to determine the labels for other spectra. An advantage of this

$\overline{{ }^{14} \text { https://github.com/andycasey/AnniesLasso }}$ 
Table 1

PW 1 Spectroscopic Results

\begin{tabular}{|c|c|c|c|c|c|c|c|c|c|c|c|c|c|c|c|c|}
\hline \multirow{2}{*}{$\begin{array}{l}\text { Name } \\
\text { PW1-00 }\end{array}$} & \multirow{2}{*}{$\begin{array}{c}\text { Gaia ID } \\
(\mathrm{J} 2000) \\
3466763224691512064\end{array}$} & \multirow{2}{*}{$\begin{array}{c}\text { R.A. } \\
11: 55: 31.8\end{array}$} & \multirow{2}{*}{$\begin{array}{c}\text { Decl. } \\
-33: 09: 11.6\end{array}$} & \multirow{2}{*}{$\begin{array}{c}G \\
15.10\end{array}$} & \multirow{2}{*}{$\frac{\begin{array}{c}G_{\mathrm{BP}}-G_{\mathrm{RP}} \\
(\mathrm{mag})\end{array}}{-0.11}$} & \multicolumn{2}{|c|}{$\begin{array}{c}\mu_{\text {R.A. }} \mu_{\left.\text {(mas } \mathrm{yr}^{-1}\right)} \\
\mu_{\text {Decl. }} .\end{array}$} & \multirow{2}{*}{$\begin{array}{l}\mathrm{S} / \mathrm{N} \\
11.8\end{array}$} & \multicolumn{2}{|c|}{$V_{\mathrm{LSR}}^{V_{\left.\mathrm{km} \mathrm{s}^{-1}\right)} \sigma_{V}}$} & \multirow{2}{*}{$\begin{array}{c}T_{\text {eff }} \\
14465\end{array}$} & \multirow{2}{*}{$\begin{array}{r}\sigma_{T_{\text {eff }}} \\
766\end{array}$} & \multicolumn{2}{|c|}{$\log _{(\mathrm{dex})} \sigma_{\log g}$} & \multicolumn{2}{|c|}{$\begin{array}{l}{[\mathrm{Fe} / \mathrm{H}]} \\
\quad(\operatorname{dex})\end{array}$} \\
\hline & & & & & & -0.58 & 0.46 & & 239.7 & 7.3 & & & 2.25 & 0.12 & -0.81 & 0.32 \\
\hline PW1-01 & 3480054567924428032 & $11: 56: 00.4$ & $-29: 28: 48.9$ & 16.30 & -0.21 & -0.45 & 0.42 & 14.2 & 257.1 & 6.7 & 17326 & 873 & 3.38 & 0.13 & -0.82 & 0.32 \\
\hline PW1-02 & 3480064910205689088 & $11: 56: 18.3$ & $-29: 19: 20.8$ & 16.39 & -0.24 & -0.85 & 0.55 & 12.4 & 255.3 & 7.3 & 16340 & 1000 & 3.20 & 0.16 & -1.06 & 0.35 \\
\hline PW1-03 & 3480053399693321984 & $11: 56: 12.0$ & $-29: 31: 44.4$ & 16.71 & -0.23 & -0.37 & 0.50 & 4.3 & 298.5 & 20.0 & 17436 & 2041 & 3.22 & 0.36 & -0.33 & 0.57 \\
\hline PW1-04 & 3486242378847130624 & $12: 00: 11.5$ & $-27: 59: 50.4$ & 16.78 & -0.17 & -0.76 & 0.45 & 8.8 & 235.1 & 11.3 & 14654 & 953 & 3.38 & 0.19 & -1.11 & 0.30 \\
\hline PW1-05 & 3479870124848931200 & $11: 57: 20.8$ & $-29: 27: 45.2$ & 16.84 & -0.20 & -0.58 & 0.55 & 16.1 & 262.7 & 5.3 & 17534 & 548 & 3.76 & 0.09 & -1.04 & 0.18 \\
\hline PW1-06 & 3479874106283037568 & $11: 56: 56.3$ & $-29: 25: 11.6$ & 16.84 & -0.20 & -0.71 & 0.57 & 29.1 & 259.0 & 3.3 & 15567 & 263 & 3.77 & 0.05 & -1.25 & 0.12 \\
\hline PW1-07 & 3480052712498583424 & $11: 55: 46.3$ & $-29: 33: 56.9$ & 16.87 & -0.24 & -0.43 & 0.50 & 30.6 & 267.9 & 2.7 & 17321 & 315 & 3.51 & 0.05 & -1.26 & 0.12 \\
\hline PW1-08 & 3486167027940903296 & $11: 56: 04.8$ & $-28: 28: 38.8$ & 16.95 & -0.21 & -0.47 & 0.59 & 32.0 & 274.9 & 2.7 & 17930 & 321 & 3.64 & 0.05 & -1.32 & 0.10 \\
\hline PW1-09 & 3480071644714348032 & $11: 55: 41.7$ & $-29: 17: 32.5$ & 17.01 & -0.23 & -0.53 & 0.46 & 29.3 & 266.9 & 3.3 & 17878 & 350 & 3.62 & 0.05 & -1.24 & 0.12 \\
\hline PW1-10 & 3479874690398598784 & $11: 57: 09.5$ & $-29: 21: 50.6$ & 17.09 & -0.18 & -0.54 & 0.40 & 27.2 & 288.7 & 4.0 & 15805 & 296 & 3.64 & 0.06 & -1.16 & 0.14 \\
\hline PW1-11 & 3485879024613971328 & $11: 57: 42.5$ & $-29: 20: 32.8$ & 17.14 & -0.12 & 0.22 & 0.42 & 22.7 & 278.2 & 4.7 & 14561 & 329 & 3.60 & 0.07 & -1.17 & 0.15 \\
\hline PW1-12 & 3480036975738300800 & $11: 53: 53.6$ & $-29: 38: 55.3$ & 17.17 & -0.17 & -0.49 & 0.47 & 23.4 & 262.4 & 4.0 & 16105 & 372 & 3.66 & 0.07 & -1.25 & 0.14 \\
\hline PW1-13 & 3467765189021964544 & 12:00:18.7 & $-30: 14: 30.8$ & 17.19 & -0.16 & -0.23 & 0.30 & 19.9 & 341.6 & 5.3 & 17216 & 451 & 4.19 & 0.07 & -1.15 & 0.17 \\
\hline PW1-14 & 3480121844292017920 & $11: 54: 23.4$ & $-29: 15: 42.0$ & 17.42 & -0.22 & -0.40 & 0.44 & 24.2 & 280.7 & 4.0 & 17254 & 401 & 3.78 & 0.06 & -1.24 & 0.14 \\
\hline PW1-15 & 3480049757560914944 & $11: 54: 25.0$ & $-29: 23: 44.4$ & 17.54 & -0.20 & -0.50 & 0.21 & 32.9 & 267.2 & 2.7 & 17472 & 283 & 3.67 & 0.05 & -1.18 & 0.09 \\
\hline PW1-16 & 3480046557809199616 & $11: 54: 46.1$ & $-29: 25: 33.5$ & 17.57 & -0.22 & -0.54 & 0.45 & 37.7 & 266.4 & 2.7 & 15726 & 225 & 3.31 & 0.04 & -1.16 & 0.11 \\
\hline PW1-17 & 3480070957519575552 & $11: 55: 27.6$ & $-29: 21: 43.8$ & 17.59 & -0.17 & -0.48 & 0.27 & 37.8 & 268.3 & 2.7 & 16894 & 235 & 3.66 & 0.04 & -1.27 & 0.08 \\
\hline PW1-18 & 3479762991184812544 & $11: 57: 32.4$ & $-30: 14: 56.6$ & 17.67 & -0.19 & -1.00 & 0.36 & 38.1 & 275.9 & 2.7 & 15871 & 218 & 3.62 & 0.04 & -1.25 & 0.08 \\
\hline PW1-19 & 3486175647939287680 & $11: 57: 29.7$ & $-28: 29: 54.7$ & 17.70 & -0.12 & -0.22 & 0.29 & 31.5 & 257.7 & 2.7 & 13979 & 224 & 3.49 & 0.05 & -1.20 & 0.10 \\
\hline PW1-20 & 3479873354664338688 & 11:57:09.4 & $-29: 25: 47.3$ & 17.69 & -0.07 & -0.61 & 0.37 & 29.8 & 244.3 & 4.0 & 13609 & 210 & 3.82 & 0.05 & -1.22 & 0.10 \\
\hline PW1-21 & 3486267147922619136 & $12: 00: 22.2$ & $-27: 56: 54.3$ & 17.74 & -0.18 & -0.43 & 0.18 & 31.6 & 289.6 & 2.7 & 16060 & 303 & 3.38 & 0.05 & -1.28 & 0.11 \\
\hline PW1-22 & 3480047141924659456 & $11: 53: 57.4$ & $-29: 30: 37.4$ & 17.75 & -0.18 & -0.40 & 0.40 & 28.5 & 277.2 & 3.3 & 15402 & 280 & 3.73 & 0.05 & -1.28 & 0.11 \\
\hline PW1-23 & 3480049856344140288 & $11: 54: 37.3$ & $-29: 22: 36.4$ & 17.80 & -0.06 & -0.53 & 0.61 & 31.9 & 284.5 & 2.7 & 15845 & 285 & 3.35 & 0.05 & -1.23 & 0.12 \\
\hline PW1-24 & 3485874897149344256 & $11: 57: 58.3$ & $-29: 24: 47.2$ & 17.79 & -0.12 & -0.51 & 0.44 & 29.9 & 257.2 & 3.3 & 15246 & 291 & 3.47 & 0.06 & -1.19 & 0.12 \\
\hline PW1-25 & 3480070510842975104 & $11: 55: 07.5$ & $-29: 21: 14.9$ & 17.82 & -0.08 & -0.71 & 0.37 & 29.3 & 271.2 & 4.0 & 13570 & 201 & 4.00 & 0.05 & -1.15 & 0.10 \\
\hline PW1-26 & 3480122325328358144 & $11: 54: 25.7$ & $-29: 13: 24.8$ & 18.30 & -0.23 & -0.42 & 0.45 & 21.3 & 292.6 & 4.7 & 14914 & 189 & 3.01 & 0.05 & -1.21 & 0.02 \\
\hline PW1-27 & 3486267251001844736 & $12: 00: 23.1$ & $-27: 55: 21.8$ & 17.94 & -0.12 & -0.89 & 0.37 & 29.3 & 270.0 & 3.3 & 15050 & 264 & 3.30 & 0.05 & -1.18 & 0.05 \\
\hline
\end{tabular}




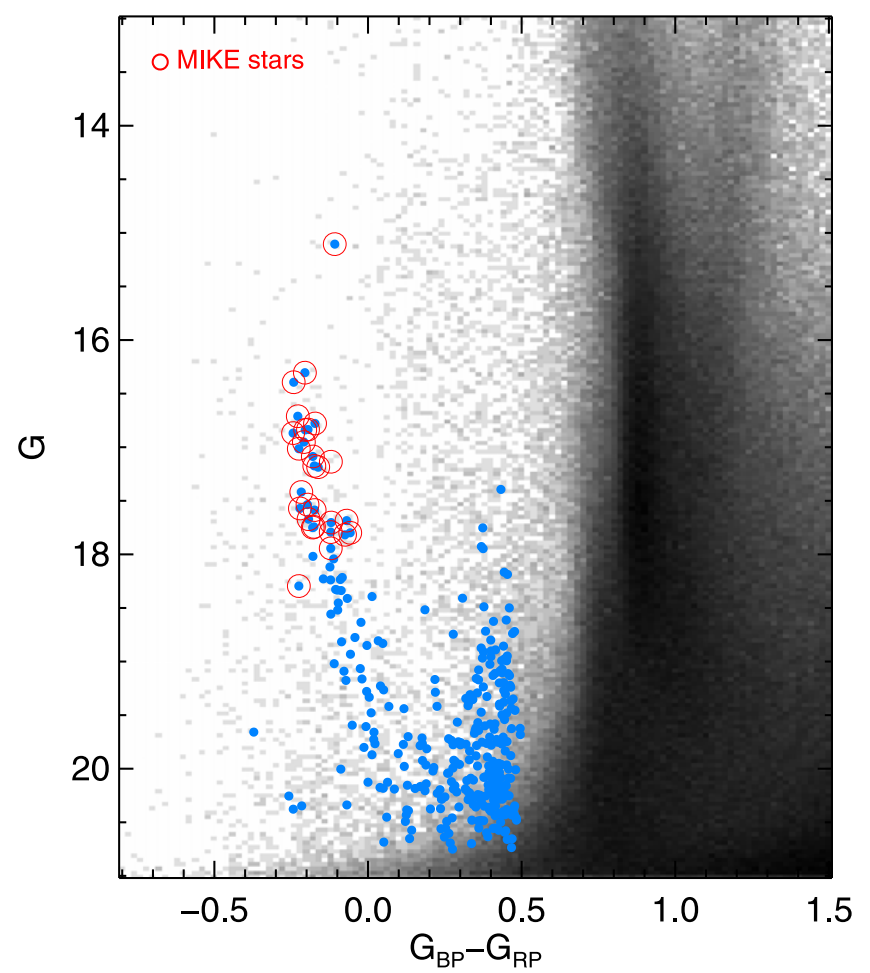

Figure 2. Color-magnitude diagram using Gaia photometry in the vicinity of PW 1 (black points); the specific spatial constraints are $173.5<\alpha<185^{\circ} .5$, $-35^{\circ} 0<\delta<-23^{\circ}$. 0 . Filled (blue) circles are probable PW 1 members (with $\left.G_{\mathrm{BP}}-G_{\mathrm{RP}}<0.5\right)$ based on the proper motion model described in Paper I. MIKE spectra were obtained for the 28 stars indicated by open red circles, including PW1-03, which was not used in the analysis due to low $\mathrm{S} / \mathrm{N}$.

method is that it exploits the information available in the spectra and is also fully automated. First, we construct a grid of 330 synthetic spectra (at a resolution of $1.5 \AA$ ) with the Synspec $^{15}$ (Hubeny \& Lanz 2011, 2017) spectral synthesis software and IDL wrappers and auxiliary scripts (A. Prieto 2019, private communication). Given an input atmospheric model, a linelist, and stellar parameters and abundances, Synspec solves the radiative transfer equation over a specified wavelength range and resolution resulting in a synthetic spectrum. We use the Kurucz LTE atmospheric models and include both atomic and molecular lines. For the spectral grid, the $T_{\text {eff }}$ ranges from $11,000 \mathrm{~K}$ to $21,000 \mathrm{~K}$ in steps of $1000 \mathrm{~K}$, the $\log g$ ranges from 2.0 to 5.0 in steps of $0.5 \mathrm{dex}$, and the $[\mathrm{Fe} / \mathrm{H}]$ ranges from -1.5 to +0.5 in steps of $0.25 \mathrm{dex}$ (the boundary of the grid is shown in the bottom panel of Figure 6). The synthetic spectra were interpolated onto the wavelength grid of the final MIKE spectra and normalized. For The Cannon model, we used a quadratic polynomial model to represent how the flux varies with the three stellar parameters at each wavelength. To provide a simple sanity check of the model generated by The Cannon, we determined the stellar parameters for the training set spectra using The Cannon model and compared these results to the training set input parameters. We found no significant biases. Specifically, the $T_{\text {eff }}$ had an offset of $0.5 \mathrm{~K}$ and scatter of $\sigma=250 \mathrm{~K}, \log g$ had a mean offset of $-0.003 \mathrm{dex}$ and $\sigma=0.06 \mathrm{dex}$, while $[\mathrm{Fe} / \mathrm{H}]$ had no offset with a scatter of $\sigma=0.12$ dex.

We use the initial stellar parameters determined with The Cannon to refine the normalization of the MIKE spectra using a

\footnotetext{
15 http://nova.astro.umd.edu/Synspec43/synspec.html
}

scaling factor determined by the ratio of the observed spectrum divided by the best-fit Cannon spectrum with heavy Gaussiansmoothing (FWHM = $38 \AA$ ). New Cannon solutions were then found for these "renormalized" observed spectra. Two example fits from The Cannon are shown in Figure 4 in comparison to their best-fit Cannon model and its parameters.

We use the emcee (Goodman \& Weare 2010; ForemanMackey et al. 2013) Markov Chain Monte Carlo (MCMC) sampler to determine the stellar parameters for each star and their uncertainties. We run with 30 walkers for 1000 steps and the first 200 are discarded as "burn-in" steps. Figure 5 shows an example "corner" plot of the posterior distributions for PW1-18 and the median values with the blue lines. Typical statistical uncertainties are $\sim 300 \mathrm{~K}$ in $T_{\text {eff }}, 0.06$ dex in $\log g$, and 0.10 dex in $[\mathrm{Fe} / \mathrm{H}]$.

Figure 6 shows the distribution of $\log g$ versus $T_{\text {eff }}$ colorcoded by $[\mathrm{Fe} / \mathrm{H}]$ and $V_{\mathrm{LSR}}$ in comparison to PARSEC isochrones. We note that one or two stars (including PW113) might be hot horizontal branch (HB) stars based on the best-fit $\log g$ and $T_{\text {eff }}$ (e.g., Zhang et al. 2019). Confirming whether a star is either on the HB or MS evolutionary stage is beyond the scope of this paper due to the lack of He abundance information. However, we emphasize that the inclusion of a few potential HB stars does not affect our dynamical analysis and main conclusions because all the stars in our sample share consistent proper motions and RVs.

Figure 7 shows the resulting $[\mathrm{Fe} / \mathrm{H}]$ distribution of $\mathrm{PW} 1$ stars, which is peaked at $[\mathrm{Fe} / \mathrm{H}] \approx-1.23$ and has a dispersion of 0.06 dex. This spectroscopically measured metallicity is in good agreement with our photometry-based metallicity measurement of $[\mathrm{Fe} / \mathrm{H}]=-1.1$ in Paper I. The small dispersion of the PW $1[\mathrm{Fe} / \mathrm{H}]$ values suggests that our uncertainties might be overestimated and that our true uncertainties are closer to $\sim 0.05$ dex. The derived stellar parameters and their uncertainties are also provided in Table 1.

\section{Results}

\subsection{PW 1 Velocity}

With proper motion and RV information for individual stars in PW 1, along with a constraint on the mean distance to PW 1 (Paper I), we construct a simple model for its internal velocity structure to infer the true mean velocity and velocity dispersion from these projected quantities. We use the $N=22$ (out of 28) stars with spectroscopic $\mathrm{S} / \mathrm{N} \geqslant 10$ for this analysis.

We assume that the $3 \mathrm{D}$ velocity of each $n$ star, $v_{n}$, is drawn from a 3D Gaussian with mean $\boldsymbol{v}_{0}$ and a diagonal covariance matrix $\boldsymbol{C}=\sigma_{v}^{2} \mathbb{I}_{3}$, where $\sigma_{v}$ is the (assumed isotropic) velocity dispersion, and $\mathbb{I}_{3}$ is the identity matrix. ${ }^{16}$ For each individual star, we only observe projected or astrometric quantities, like sky position $(\alpha, \delta)$, distance $d$, proper motions $\left(\mu_{\alpha}, \mu_{\delta}\right)$, and RV $v_{r}$. We assume that the sky position of each star is known with infinite precision, the observed distance is unresolved and given by the mean cluster distance and uncertainty (Paper I), the proper motions are given by Gaia with a 2D covariance matrix $\boldsymbol{C}_{\mu}$, and the RVs are measured in this work. The (unobserved) true 3D velocity of each star, drawn from the model given above, is related to the observed astrometric quantities through a projection matrix that depends on sky

\footnotetext{
${ }^{16}$ We also tried allowing generic velocity anisotropies (non-diagonal $\boldsymbol{C}$ ) but found that the resulting matrix was consistent with being isotropic within the derived uncertainties.
} 


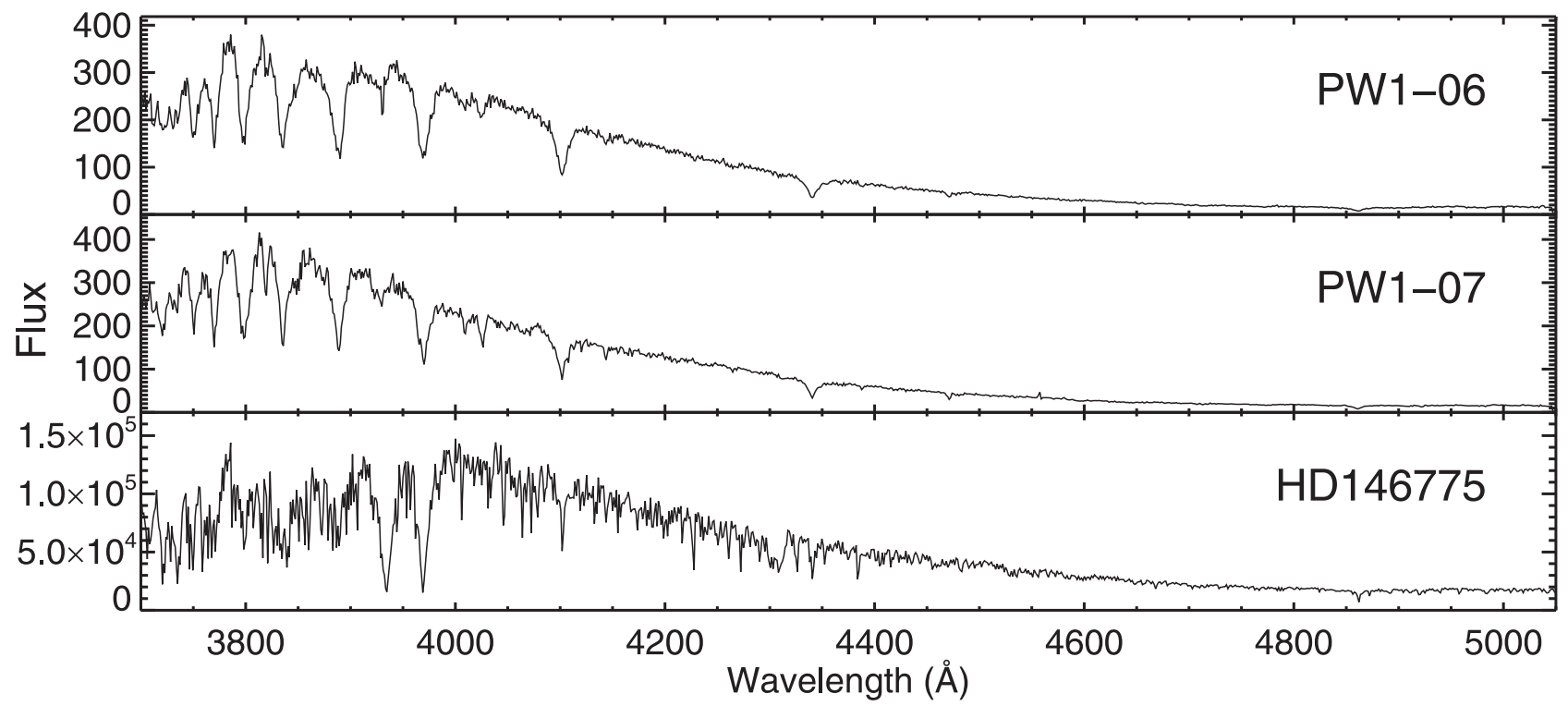

Figure 3. Three example MIKE blue-arm spectra. The top two panels show example PW 1 stars, PW1-06 (S/N = 29) and PW1-07 (S/N = 30), while the bottom panel is the reference star HD146775 $(\mathrm{S} / \mathrm{N}=826)$.

(The data used to create this figure are available.)

position (see, e.g., the appendix of Oh et al. 2017). The full model therefore has $N+3 N+3+1=92$ parameters: the true distance to each star, the true 3D velocity vector of each star, the mean velocity of PW 1 , and the velocity dispersion. This model is implemented in the Stan (Carpenter et al. 2017) probabilistic programming language, and we use the built-in No-U-Turn Hamiltonian Monte Carlo sampler (Homan \& Gelman 2014) to generate posterior samples over all of the model parameters. We run the sampler for 4000 steps in total for four independent Markov Chains: 2000 burn-in and tuning steps (that are discarded), and then a further 2000 steps for each chain, from which we assess convergence by computing the effective sample size and Gelman-Rubin convergence statistic for each chain.

Given the posterior samples generated as described above, we measure a mean barycentric RV for PW 1 of $276.7 \pm$ $2.5 \mathrm{~km} \mathrm{~s}^{-1}$ and mean proper motion of $(-0.52,0.42) \pm$ $(0.04,0.03)$ mas $^{-1}$. Assuming a total solar velocity of $(11.1,220+12.24,7.25) \mathrm{km} \mathrm{s}^{-1}$ (Schönrich et al. 2010), this corresponds to a Galactocentric (Cartesian) velocity for PW 1 of $\boldsymbol{v}_{\text {galcen }} \approx(0.6,2.2,186) \mathrm{km} \mathrm{s}^{-1}$. We find a velocity dispersion of $\sigma_{v}=11.0 \pm 2 \mathrm{~km} \mathrm{~s}^{-1}$, which is consistent with a robust standard deviation computed from the RVs alone (Section 3.1), indicating that the constraint on the velocity dispersion comes predominantly from the RV data in this work. Figure 8 shows the individual RV measurements of each observed PW 1 star (black points) as a function of R.A., along with the inferred mean velocity (blue) and velocity dispersion (orange).

In the sections to follow, we compare the inferred LOS velocity of PW 1 with $\mathrm{H}$ I radio data (e.g., from GASS) where it is necessary to convert to the "kinematic local standard of rest," which historically uses the average of solar neighborhood star velocities (Delhaye 1965; Gordon 1976). ${ }^{17}$ In this reference frame, the mean velocity is $V_{\mathrm{LSR}}=273.4 \pm 2.5 \mathrm{~km} \mathrm{~s}^{-1}$.

\footnotetext{
17 The solar motion is assumed to be $20.0 \mathrm{~km} \mathrm{~s}^{-1}$ toward $\left(18 \mathrm{hr},+30^{\circ}\right)$ at epoch 1900.0.
}

\subsection{LA Origin}

Figure 9 shows the position-velocity diagram of the PW 1 stars and the H I gas (GASS; McClure-Griffiths et al. 2009) using the Gaussian decomposition techniques described in Nidever et al. (2008). The mean $V_{\mathrm{LSR}}$ velocity of $273.4 \mathrm{~km} \mathrm{~s}^{-1}$ of the PW 1 stars is very similar to the velocity of the LA II gas of $233 \mathrm{~km} \mathrm{~s}^{-1}$. Absorption-line studies of the LA HI find a value of $[\mathrm{Fe} / \mathrm{H}] \sim$ -1 dex (Lu et al. 1998; Fox et al. 2018; Richter et al. 2018), which is similar to the mean metallicity of PW 1 stars studied here of $[\mathrm{Fe} / \mathrm{H}] \sim-1.23 \mathrm{dex}$ (see Figure 7 ). Therefore, we conclude that the PW 1 stars are physically associated with the LA II gas based on their similar RVs and metallicities.

\subsection{Spatial Offset between Stars and Gas}

The current location of PW 1 is close to LA II on the sky, but there is little gas in its immediate vicinity in the positionvelocity diagram (Figure 9). The top panel of Figure 10 shows the spatial offset between PW 1 and the LA II gas in the MS coordinate system $\left(L_{\mathrm{MS}}, B_{\mathrm{MS}}\right)$. The gas from which PW 1 originated should experience ram pressure from the MW hot halo gas (e.g., Mastropietro et al. 2005). Because the stars will not feel this force, the gas and stars will decouple over time with the net effect that the gas trails the stars in their orbit. With this in mind, it is not surprising that there is little gas in the immediate vicinity of PW 1 . Thus, the spatial offset does not rule out LA II as the origin gas for PW 1.

Quantitatively, the magnitude of the spatial decoupling between the stars and gas is determined by a $2 \mathrm{D}$ crosscorrelation of the star and gas maps (Figure 10, top). The individual maps were masked such that only the highestdensity regions were used. The result of the cross-correlation is in the middle panel of Figure 10. The peak of the crosscorrelation is at an offset of $\left(-10^{\circ} .15,+1.44\right)$ in $\left(L_{\mathrm{MS}}, B_{\mathrm{MS}}\right)$, which is indicated as a red cross in the middle panel of Figure 10. The bottom panel of Figure 10 shows that shifted PW 1 positions are well aligned with the high-density peaks of the $\mathrm{H}$ I gas. These shift positions are more representative of the 


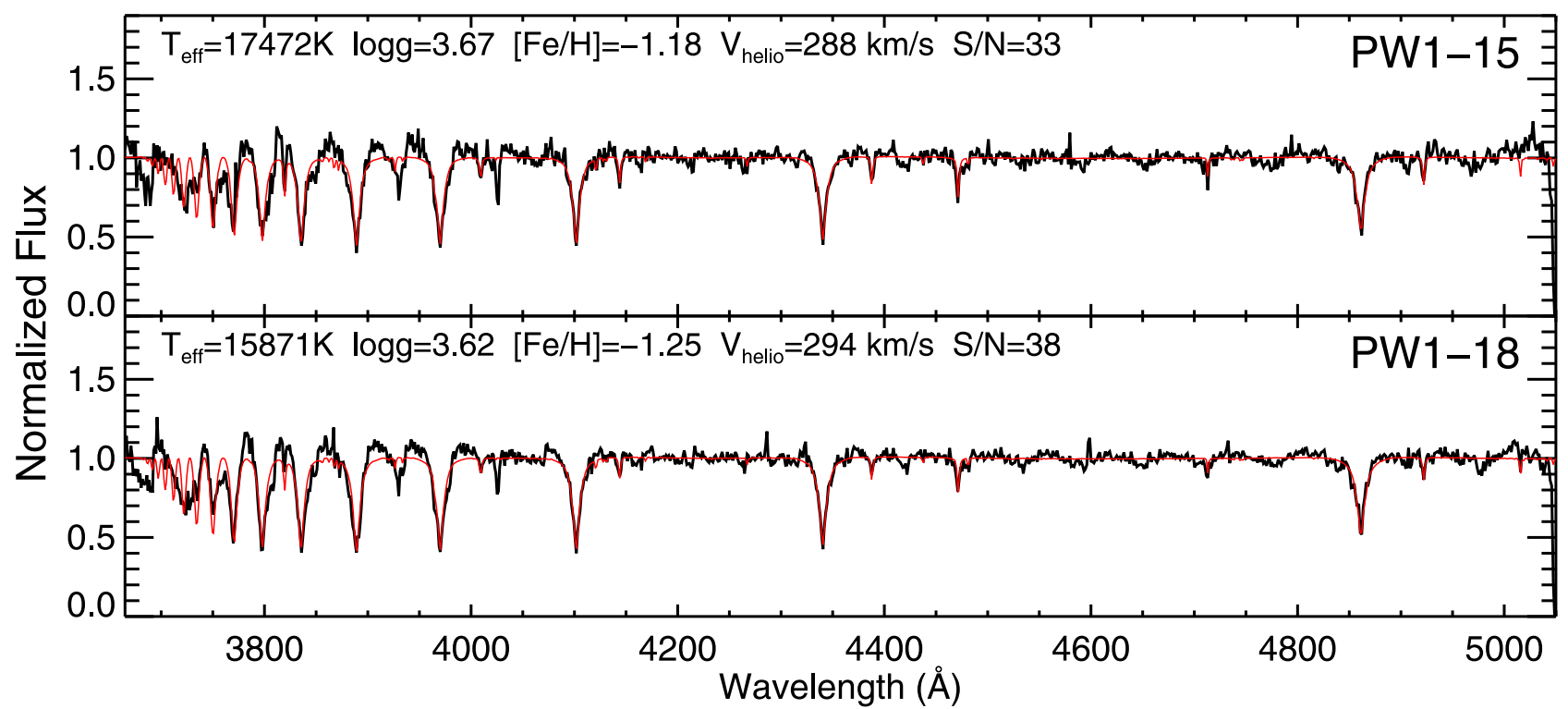

Figure 4. Example best-fit spectra from The Cannon. MIKE spectra for PW1-15 and PW1-18 (black) are shown with the best-fit Cannon models overlaid (red). These show excellent agreement. The best-fit stellar and RV parameters are noted in the top left corner for each star.

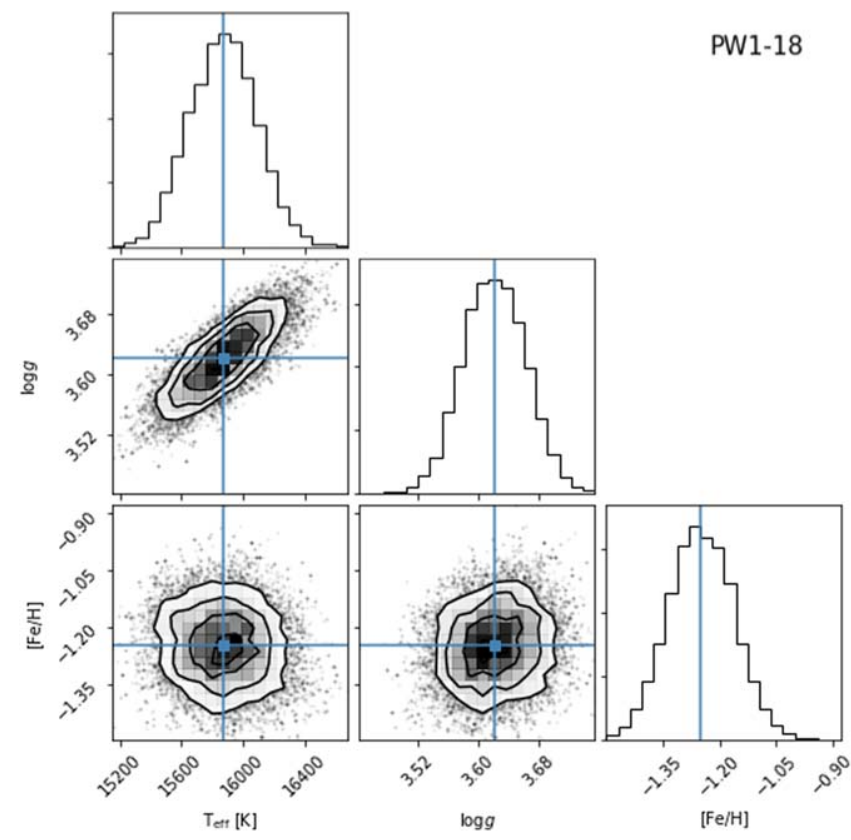

Figure 5. Example corner plot showing the emcee MCMC posterior distributions for PW1-18. The median values are shown by the blue lines.

original relative positioning of the gas forming PW 1 within the LA II complex.

Figure 11 shows a slightly different visualization of this process. The top panel of Figure 11 zooms into the $\left(L_{\mathrm{MS}}, B_{\mathrm{MS}}\right)$ region around the position-shifted stars with $V_{\mathrm{LSR}}$ measurements. Ram pressure will also act to modify the velocities of the gas it acts on, and thus an exact match between the velocities of the birth gas and resulting stars is not expected. The bottom panel of Figure 11 is the resulting velocity-position diagram; a $30 \mathrm{~km} \mathrm{~s}^{-1}$ velocity offset is required to have the PW 1 stars align with the densest portions of the gas in position-velocity space. We will use this offset in the next section to place a constraint on the halo gas driving the ram pressure effects.

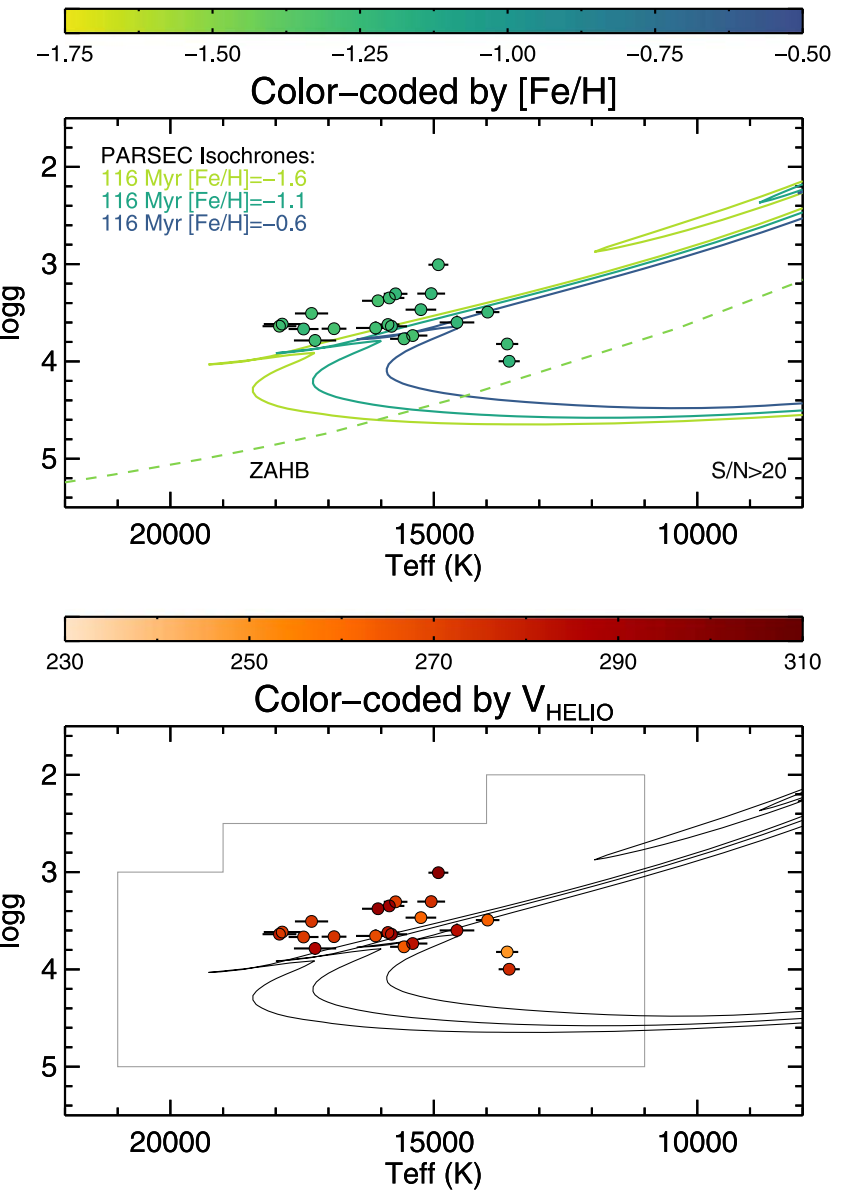

Figure 6. Stellar parameters determined from The Cannon for the 21 PW 1 stars with $\mathrm{S} / \mathrm{N} \geqslant 20$ and compared to PARSEC isochrones. The top panel shows $\log g$ vs. $T_{\text {eff }}$ color-coded by $[\mathrm{Fe} / \mathrm{H}]$, whereas the bottom panel shows the same points color-coded by $V_{\text {hel }}$. As indicated by color coding in the top panel, the isochrones have an age of $116 \mathrm{Myr}$ and a metallicity of $[\mathrm{Fe} /$ $\mathrm{H}]=-1.6,-1.1$, and -0.6 dex, respectively. The $[\mathrm{Fe} / \mathrm{H}]=-1.48$ zero-age horizontal branch (ZAHB) from Dorman et al. (1993) is also shown as a dashed line. The boundary of the synthetic spectral grid used in The Cannon analysis is shown in light gray in the bottom panel. 


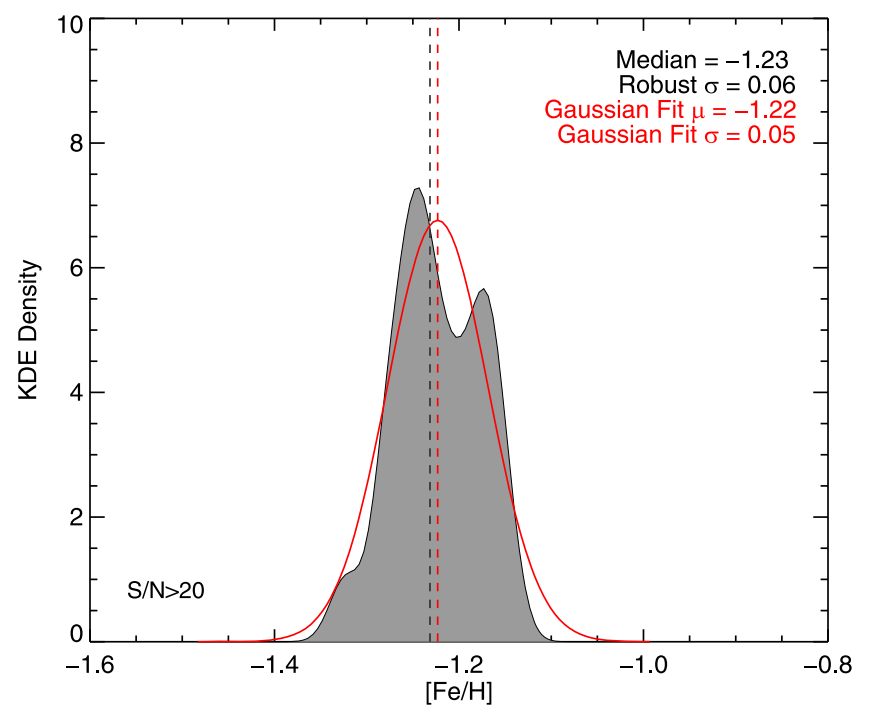

Figure 7. Kernel density estimate $(\mathrm{KDE})$ of the distribution of $[\mathrm{Fe} / \mathrm{H}]$ measurements for $21 \mathrm{PW} 1$ stars with $\mathrm{S} / \mathrm{N} \geqslant 20$ and a bandwidth of $0.04 \mathrm{dex}$, which is smaller than the typical $[\mathrm{Fe} / \mathrm{H}]$ uncertainty of $\sim 0.10$ dex. A single Gaussian model is fit to the histogram and has a peak at -1.22 and $\sigma$ of 0.05 dex. The median $[\mathrm{Fe} / \mathrm{H}]$ is -1.23 ; the standard deviation is 0.06 dex.
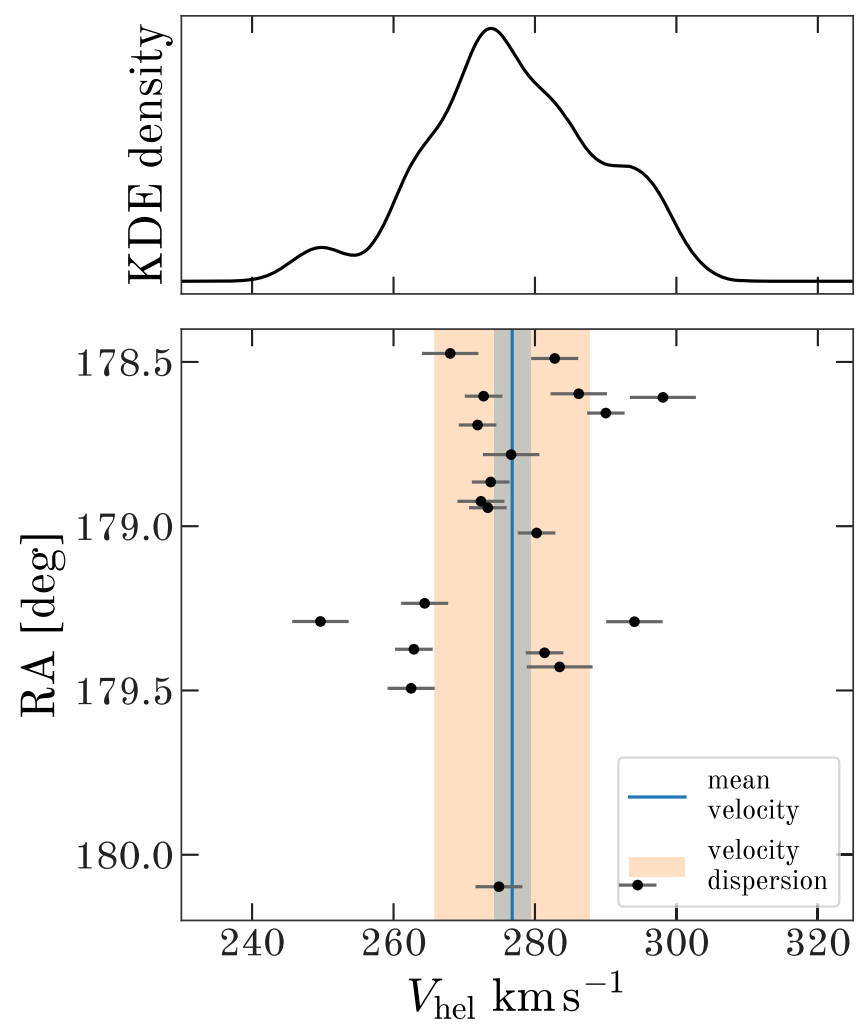

Figure 8. Top panel: kernel density estimate of the distribution of RV measurements with a bandwidth $b=3 \mathrm{~km} \mathrm{~s}^{-1}$. Bottom panel: individual RV measurements for PW 1 stars with uncertainties (black markers and error bars) as a function of R.A. The solid line (blue) shows the inferred mean RV of PW 1, and the shaded (blue) region shows the uncertainty on the mean RV, $276.7 \pm 2.5 \mathrm{~km} \mathrm{~s}^{-1}$. The larger shaded region (orange) shows the median posterior value of the velocity dispersion of PW $1,11.0 \pm 2 \mathrm{~km} \mathrm{~s}^{-1}$.

\subsection{Ram Pressure}

If the spatial and velocity offsets between this gas and PW 1 are indeed due to a roughly constant ram pressure, then the measured position-velocity offsets become a means of inferring the properties of the gas performing the ram pressure. In particular, we place a constraint on the average density of the "hot" MW-halo gas, a medium that remains difficult to characterize directly (for an overview see Putman et al. 2012).

At a distance of $29 \mathrm{kpc}$, the angular offset of $10^{\circ} .15$ corresponds to a tangential distance of $\sim 5.14 \mathrm{kpc}$. Over $116 \mathrm{Myr}$ that corresponds to a mean "drift" velocity of $\sim 43.3 \mathrm{~km} \mathrm{~s}^{-1}$ or a deceleration of $\sim 0.747 \mathrm{~km} \mathrm{~s}^{-1} \mathrm{Myr}^{-1}$. The total change in the tangential velocity of the gas over this period is $\sim 86.6 \mathrm{~km} \mathrm{~s}^{-1}$. The $V_{\mathrm{LSR}}$ of LA II "tip" is lower than that of PW 1 by $\sim 30 \mathrm{~km} \mathrm{~s}^{-1}$. The smaller $V_{\mathrm{LSR}}$ shift would indicate that a significant fraction of the deceleration occurred in the tangential direction.

If we assume that ram pressure is the main factor in causing the gas and stars to drift apart and that this is dominated by a roughly constant ram pressure from the MW hot halo, then we can estimate the density of the MW hot halo gas as follows. The ram pressure experienced by the PW 1 "birth cloud" (BC) in LA II is defined as

$$
P=\rho_{\mathrm{MW}} v_{\mathrm{BC}}^{2}
$$

where $\rho_{\mathrm{MW}}$ is the density of the MW halo gas and $v_{\mathrm{BC}}$ is the $\mathrm{BC}$ velocity through the medium. If $l_{\mathrm{BC}}$ is the approximate diameter of the $\mathrm{BC}$, then the acceleration that it experiences $\left(a_{\mathrm{BC}}\right)$ is

$$
a_{\mathrm{BC}} \approx \frac{\rho_{\mathrm{MW}} v_{\mathrm{BC}}^{2} l_{\mathrm{BC}}^{2}}{\rho_{\mathrm{BC}} l_{\mathrm{BC}}^{3}} \approx \frac{\rho_{\mathrm{MW}} v_{\mathrm{BC}}^{2}}{\rho_{\mathrm{BC}} l_{\mathrm{BC}}} .
$$

Solving Equation (2) for the ratio of gas density between the $\mathrm{MW}$ and the $\mathrm{BC}$ gives

$$
\frac{\rho_{\mathrm{MW}}}{\rho_{\mathrm{BC}}} \approx \frac{a_{\mathrm{BC}} l_{\mathrm{BC}}}{v_{\mathrm{BC}}^{2}} .
$$

If we assume that the $\mathrm{BC}$ was undergoing a roughly constant deceleration due to ram pressure since forming PW 1, then we can estimate this deceleration from the age of PW $1(\Delta t=116 \mathrm{Myr})$ and the spatial offset $\left(\Delta x=10^{\circ} .15=5.14 \mathrm{kpc}\right)$, as

$$
a_{\mathrm{BC}} \approx \frac{2 \Delta x}{\Delta t^{2}}
$$

This results in $a_{\mathrm{BC}} \approx 0.747 \mathrm{~km} \mathrm{~s}^{-1} \mathrm{Myr}^{-1}$. The angular width (FWHM in $B_{\mathrm{MS}}$ ) of the LA II PW 1 BC from GASS is 0.75 or $0.38 \mathrm{kpc}$. Using this as an approximation of the diameter of the $\mathrm{BC}\left(l_{\mathrm{BC}}\right)$ and $v_{\mathrm{BC}} \approx 235 \mathrm{~km} \mathrm{~s}^{-1}$, we obtain $\rho_{\mathrm{MW}} / \rho_{\mathrm{BC}} \approx 0.00505$ or that the MW gas density is $\sim 0.5 \%$ of that of the PW 1 BC.

We measure the average current column density of the PW 1 BC from the H I GASS data as $N_{\mathrm{H} \text { I }} \approx 1.5 \times 10^{20}$ atoms cm ${ }^{-2}$. Assuming a distance of $29 \mathrm{kpc}$ and a width of $\sim 0.38 \mathrm{kpc}$ gives a number density of $n_{\mathrm{BC}} \approx 0.128$ atoms cm $\mathrm{cm}^{-3}$.

Finally, we derive the number density of the hot MW halo as $n_{\mathrm{MW}} \approx 6.46 \times 10^{-4}$ atoms $\mathrm{cm}^{-3}$. This rough estimate of the hot MW halo gas density is an order of magnitude higher than that predicted by the Miller \& Bregman (2013) model, which gives $\sim 4 \times 10^{-5}$ atoms cm$~^{-3}$ at this location. However, our estimate here is too simplistic for a number of reasons: (i) we are seeing an integrated effect over the orbit of PW 1, which has passed through higher densities closer to the midplane, and (ii) we consider only a single medium - the hot halocompletely ignoring the impact of the midplane and outer gas disk itself. That this simple estimate is within an order of 


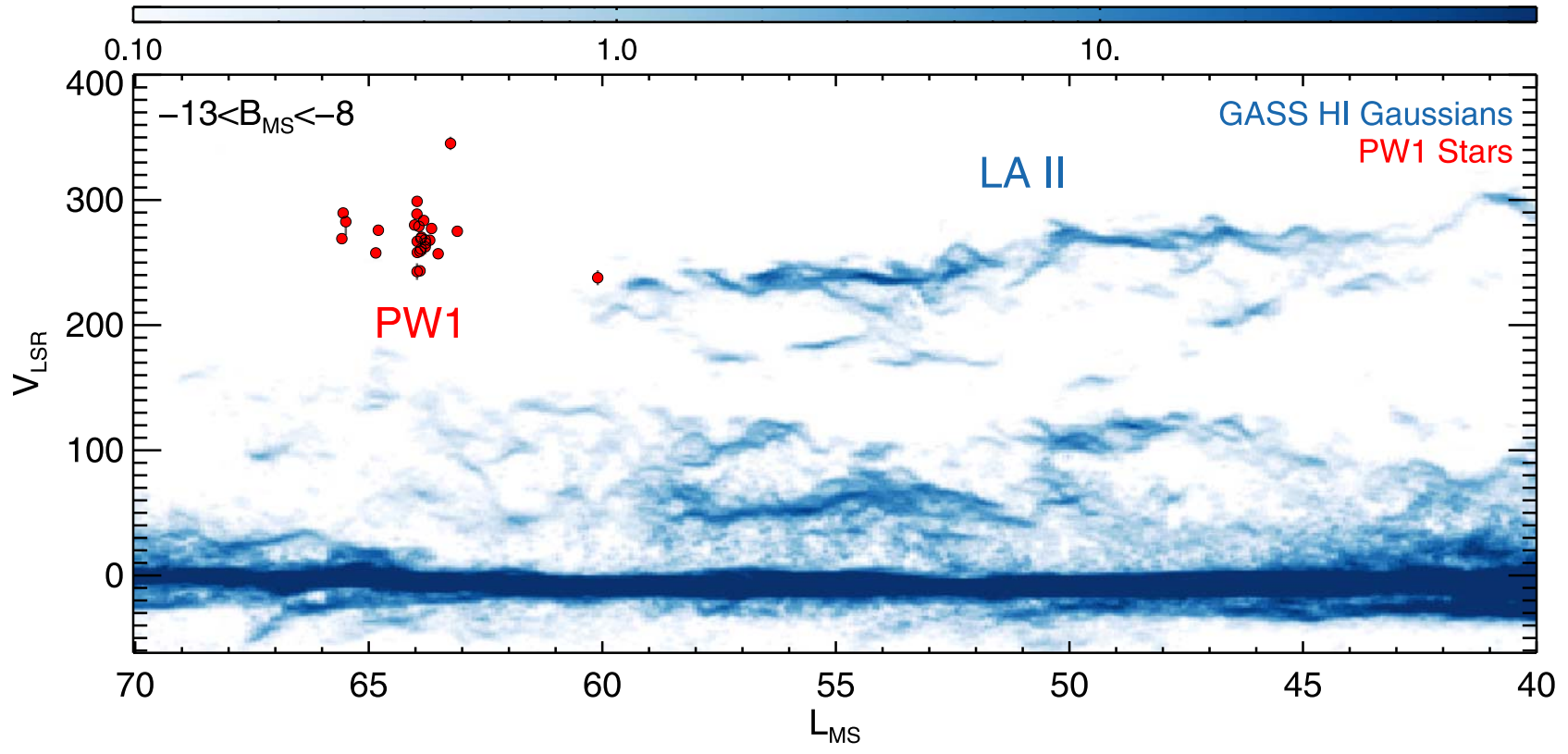

Figure 9. Position-velocity diagram of the PW 1 stars compared to the GASS H I data (McClure-Griffiths et al. 2009). The background bluescale image is the integrated intensity of the GASS Gaussian centers summed along $B_{\mathrm{MS}}$ in units of $10^{18}$ atoms cm $\mathrm{cm}^{-2}$. The tip of LA II has a velocity of $\sim 233 \mathrm{~km} \mathrm{~s}{ }^{-1}$. Each PW $1 \mathrm{star}$ is plotted at its determined $V_{\mathrm{LSR}}$ as a red filled circle with its uncertainty. The stars have a mean velocity of $\sim 273.4 \mathrm{~km} \mathrm{~s}^{-1}$, slightly higher than but similar to the gas in LA II.

magnitude of state-of-the art measurements motivates the more complex and nuanced simulations that are presented in the next subsection.

\subsection{Ram Pressure Orbit Analysis}

The offsets in sky position and velocity between the PW 1 stars and the LA II gas suggest that the gas was likely subject to a drag force or dissipated orbital energy: the offset in position is primarily along the direction of motion, and the gas velocity is slightly lower than that of PW 1 . Moreover, the order-of-magnitude computations presented in the previous subsection suggest that ram pressure from the hot halo produce effects on par with what is seen, albeit more complex interactions of the midplane are ignored. We therefore perform a set of orbit integrations to see if the observed positionvelocity offsets can plausibly be explained by gas drag from the MW halo when the full orbital motion and influence of the midplane are included.

We use the MW mass model implemented in gala (Bovy 2015; Price-Whelan 2017) as the background gravitational potential, and include a time-dependent mass component to represent the LMC. In detail, we represent the mass distribution of the LMC using a Hernquist profile (Hernquist 1990), which generally follows the the approach used in Erkal et al. (2019), except that we use a total mass of $M_{\mathrm{LMC}}=2.5 \times 10^{11} M_{\odot}$. (Laporte et al. 2018). We compute the position of the LMC at any given time by integrating the orbit of the LMC center-of-mass backward from its present-day phase-space position (using initial conditions from Patel et al. 2017). We neglect any back-reaction or response of the MW to the presence of the LMC. We use this time-dependent mass model along with the measured position and velocity of PW 1 to numerically integrate the orbit of the PW 1 backward in time from present day $\left(t_{2}=0\right)$ to its birth time $\left(t_{1}=-\tau=-116 \mathrm{Myr}\right)$. We use the Dormand-Prince eighthorder Runge-Kutta scheme (Dormand \& Prince 1980) to numerically integrate these orbits.
Once the phase-space coordinates of PW 1 at its birth time are estimated, we then integrate forward in time, now including the effects of gas drag and momentum coupling between the MW disk and the LA II gas as it crosses the Galactic midplane. We compute the drag acceleration on the gas as

$$
\boldsymbol{a}_{\mathrm{drag}}=n_{\mathrm{MW}}(\boldsymbol{x})|\boldsymbol{v}|^{2} \Sigma_{\mathrm{LA}}^{-1} \times-\frac{\boldsymbol{v}}{|\boldsymbol{v}|},
$$

where $n_{\mathrm{MW}}(\boldsymbol{x})$ is the number density of MW gas at position $\boldsymbol{x}, \boldsymbol{v}$ is the orbital velocity, and $\Sigma_{\mathrm{LA}}$ is the surface density of the LA gas (following, e.g., Vollmer et al. 2001). For the gas density, we use the gaseous halo model from Miller \& Bregman (2013) and the disk model from Kalberla \& Kerp (2009) with a Gaussian density profile in the height above the midplane $z$ such that

$$
\begin{gathered}
n_{\mathrm{MW}}(\boldsymbol{x})=n_{\text {halo }}(r)+n_{\text {disk }}(R, z), \\
n_{\text {halo }}(r)=n_{0, h}\left[1+\left(\frac{r}{r_{c}}\right)^{2}\right]^{-3 \beta / 2}, \\
n_{\text {disk }}(R, z)=n_{0, d} e^{-\frac{R-R_{\odot}}{R_{n}}} e^{-\frac{1}{2} \frac{z^{2}}{\sigma_{z}(R)^{2}}}, \\
\sigma_{z}(R)=0.85 h_{0} e^{\frac{R-R_{\odot}}{R_{0}}},
\end{gathered}
$$

where $r$ is the spherical radius, $R$ is the cylindrical radius, $R_{\odot}=8.1 \mathrm{kpc}$ is the solar Galactocentric radius, and all parameter values are taken from Kalberla \& Kerp (2009). We assume that the surface density of the PW 1 BC in LA II starts with $\Sigma_{0} \approx 50 M_{\odot} \mathrm{pc}^{-2}$ (comparable to other LMC molecular clouds; Wong et al. 2011) and ends (at present day) with a surface density equal to the measured column density of the LA II region, $\Sigma_{f} \approx 0.56 M_{\odot} \mathrm{pc}^{-2}$, such that

$$
\Sigma_{\mathrm{LA}}(t)=\exp \left[\frac{\left(\ln \Sigma_{f}-\ln \Sigma_{0}\right)}{\tau} t+\ln \Sigma_{f}\right]
$$



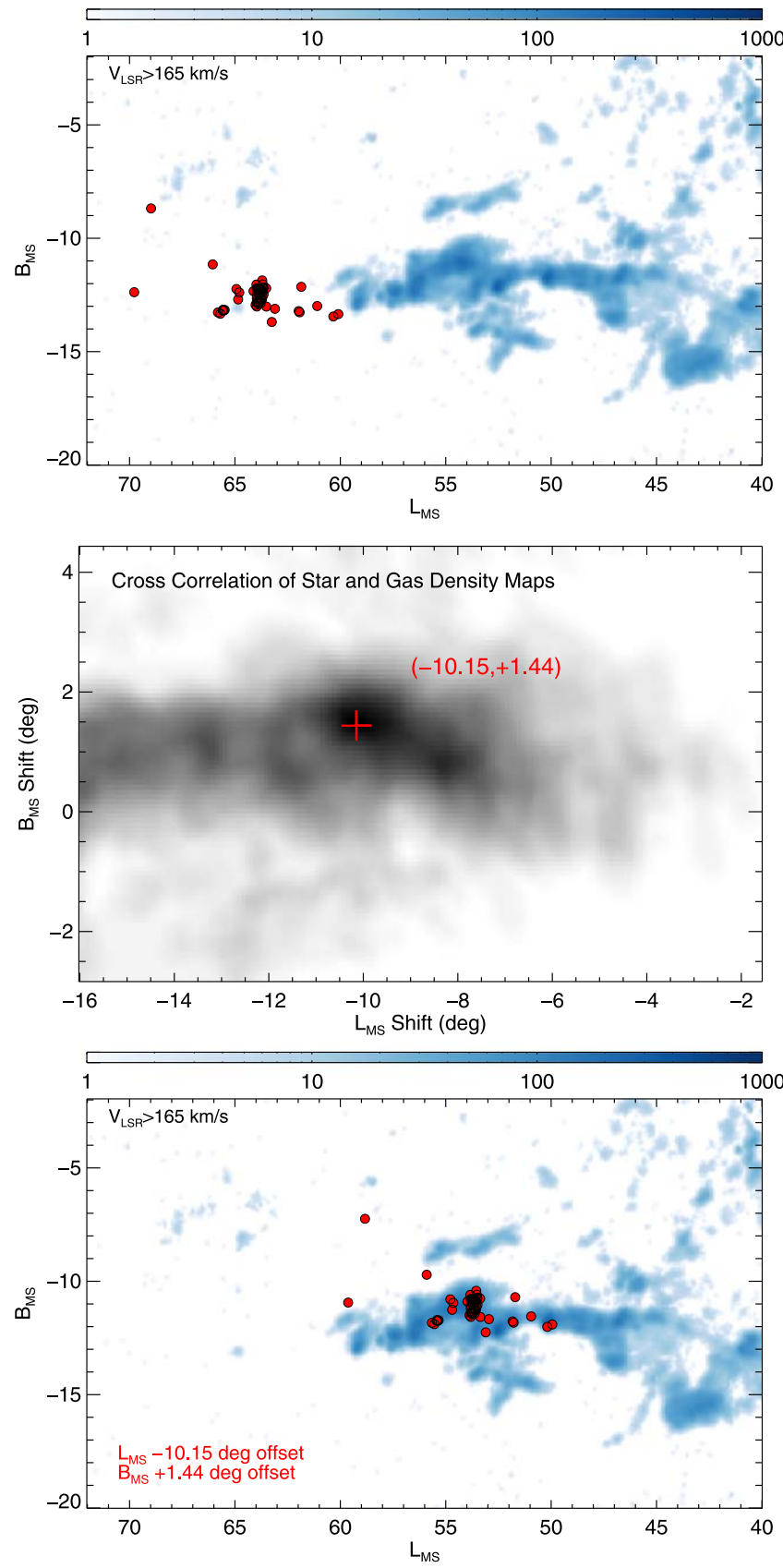

Figure 10. Spatial distribution of the $\mathrm{HI}$ gas and $71 \mathrm{PW} 1$ candidate stars based on photometry and Gaia DR2 proper motions. (top) The gas and PW 1 stars at their current ( $\left.L_{\mathrm{MS}}, B_{\mathrm{MS}}\right)$ positions. (middle) The $2 \mathrm{D}$ cross-correlation map of the star and gas density maps with the shift of $(-10.15,+1.44)$ in $\left(L_{\mathrm{MS}}, B_{\mathrm{MS}}\right)$. (Bottom) PW 1 shifted by the offset determined in the crosscorrelation.

where again $\tau$ is the age of PW $1, t_{1}=-\tau$, and $t_{2}=0$.

As mentioned above, we also allow for momentum coupling between the MW disk and the LA II gas as it passes through the Galactic midplane. To take this momentum coupling into account, we add an additional acceleration to the orbit integration, $\boldsymbol{a}_{\text {coupling, }}$, defined to point in the direction of Galactic rotation such that

$$
\boldsymbol{a}_{\text {coupling }}=\alpha \hat{\boldsymbol{v}}_{\text {rot }}(\boldsymbol{x}) e^{-\frac{1}{2} \frac{z^{2}}{\sigma_{z}(R)}},
$$

where $\alpha$ is a free parameter that determines the magnitude of the coupling, $\hat{v}_{\text {rot }}(\boldsymbol{x})$ is a unit vector that points in the direction
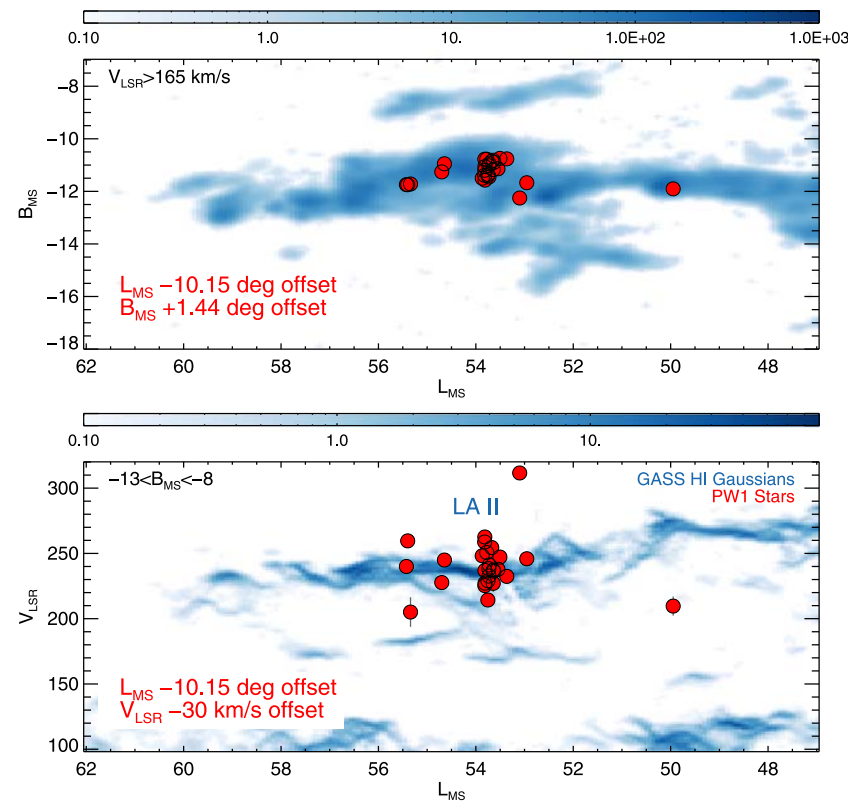

Figure 11. Spatial and position-velocity diagram for the 28 PW 1 stars with radial velocities and LA II gas. The top panel shows the positions of the PW 1 stars (red filled circles) and the LA II gas after the cross-correlation offset has been applied (see Figure 10). The bottom panel shows the same spatially offset stars in position-velocity space. A $V_{\mathrm{LSR}}$ offset of $30 \mathrm{~km} \mathrm{~s}^{-1}$ has been applied such that the stars align with the densest portions of the LA II gas.

of Galactic rotation at the position $\boldsymbol{x}$, and the Gaussian in height, $z$, makes this operate only when the gas orbit is close to the Galactic plane.

We next allow the scale densities, $n_{0, h}$ and $n_{0, d}$, and the momentum coupling parameter, $\alpha$, to vary and fit for the values of these parameters that best reproduce the observed position and velocity offsets between PW 1 and the LA II gas. We define a fiducial point in $L_{\mathrm{MS}}, B_{\mathrm{MS}}, V_{\text {hel }}$ to set the present-day location of the densest LA II gas (see Section 4.3) that could have plausibly formed PW 1:

$$
\begin{gathered}
L_{\mathrm{MS}}=53.7, \\
B_{\mathrm{MS}}=-11^{\circ} 1, \\
V_{\mathrm{hel}}=239 \mathrm{~km} \mathrm{~s}^{-1} .
\end{gathered}
$$

We construct a likelihood function using the above orbit integration scheme and evaluate the likelihood of the presentday (i.e., final) orbit phase-space coordinates relative to the fiducial point defined previously. We assume a Gaussian tolerance of $\sigma_{\mathrm{LB}}=0.5$ for $L_{\mathrm{MS}}$ and $B_{\mathrm{MS}}$, and $\sigma_{v}=1 \mathrm{~km} \mathrm{~s}^{-1}$ for $V_{\text {hel }}$ and evaluate the likelihood as

$$
\begin{aligned}
3 p\left(L_{\mathrm{MS}}, B_{\mathrm{MS}},\right. & \left.V_{\text {hel }} \mid n_{0, h}, n_{0, d}, \alpha\right) \\
= & \mathcal{N}\left(\mathrm{L}_{\text {orbit }} \mid L_{\mathrm{MS}}, \sigma_{\mathrm{LB}}^{2}\right) \\
& \times \mathcal{N}\left(\mathrm{B}_{\text {orbit }} \mid B_{\mathrm{MS}}, \sigma_{\mathrm{LB}}^{2}\right) \\
& \times \mathcal{N}\left(V_{\text {orbit }} \mid V_{\text {hel }}, \sigma_{v}^{2}\right),
\end{aligned}
$$

where $\mathcal{N}\left(\cdot \mid \mu, \sigma^{2}\right)$ represents the normal distribution with mean $\mu$ and variance $\sigma^{2}$. In practice, we implement this function (programmatically) over the $\log$ values of the three parameters (because they must be positive), but we assume uniform priors in the parameter values over the domain ( $a$, $b)=\left(e^{-30}, e^{5}\right)$ for each parameter such that the prior 

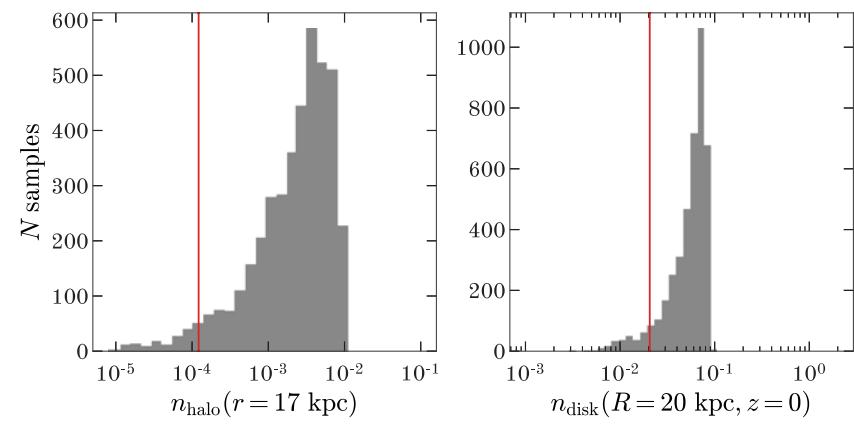

Figure 12. Posterior samples from the ram pressure orbit analysis, transformed to values of the inferred halo gas density at $r=17 \mathrm{kpc} n_{\text {halo }}(17)$ (left panel) and disk gas density (right panel) at the midplane at $(R, z)=(20,0) \mathrm{kpc}$, $n_{\text {disk }}(20,0)$. Vertical red lines show the values from the default MW model from Miller \& Bregman (2013) and Kalberla \& Kerp (2009).

distribution is

$$
p\left(n_{0, h}, n_{0, d}, \alpha\right)=\mathcal{U}\left(n_{0, h} \mid a, b\right) \mathcal{U}\left(n_{0, d} \mid a, b\right) \mathcal{U}(\alpha \mid a, b),
$$

where $\mathcal{U}(\cdot \mid a, b)$ is the uniform distribution defined over the domain $(a, b)$.

We first optimize the log-posterior,

$$
\begin{aligned}
\ln p\left(n_{0, h}, n_{0, d}, \alpha \mid L_{\mathrm{MS}}, B_{\mathrm{MS}}, V_{\mathrm{hel}}\right) \\
\quad \propto \ln p\left(L_{\mathrm{MS}}, B_{\mathrm{MS}}, V_{\mathrm{hel}} \mid n_{0, h}, n_{0, d}, \alpha\right) \\
\quad+\ln p\left(n_{0, h}, n_{0, d}, \alpha\right),
\end{aligned}
$$

using the BFGS algorithm (implemented in scipy; Byrd et al. 1995; Jones et al. 2001) and then we use these optimal parameter values as initial conditions to run an MCMC sampling of the posterior probability distribution (pdf) of our parameters. We use an affine-invariant, ensemble MCMC sampler (emcee; Goodman \& Weare 2010; Foreman-Mackey et al. 2013); we run with 64 walkers for 512 "burn-in" steps (that are discarded) and then run for an additional 1024 steps. We thin the resulting chains by taking every fourth step, and combine the parameter samplings from all thinned chains. Figure 12 shows histograms of posterior samples transformed into values of the halo gas density evaluated at the orbital pericenter, $n_{\text {halo }}(17 \mathrm{kpc})$, and the disk gas density at the midplane at $n_{\text {disk }}(20 \mathrm{kpc}, 0)$. The posterior values of the coupling coefficient, $\alpha$, were all $<e^{-12}$ and thus consistent with zero. Figure 13 shows orbits drawn from the MCMC posterior distribution of the parameters compared to the $\mathrm{H} \mathrm{I}$ gas and the PW 1 birth cloud.

The best-fit parameters require a somewhat larger halo and disk gas densities than the fiducial MW gas density models from Miller \& Bregman (2013) and Kalberla \& Kerp (2009). In detail, we find

$$
\begin{gathered}
n_{\text {halo }}(17 \mathrm{kpc})=2.7_{-2.0}^{+3.4} \times 10^{-3} \text { atoms cm } \mathrm{cm}^{-3}, \\
n_{\text {disk }}(20 \mathrm{kpc}, 0)=6.0_{-2.0}^{+1.5} \times 10^{-2} \text { atoms cm } \mathrm{cm}^{-3},
\end{gathered}
$$

as compared to the fiducial values $n_{\text {halo, } \mathrm{M} 13}(17 \mathrm{kpc})=$ $1.2 \times 10^{-4}$ atoms $\mathrm{cm}^{-3}$ and $n_{\text {disk, K09 }}(20 \mathrm{kpc}, 0)=2 \times 10^{-2}$ atoms $\mathrm{cm}^{-3}$. However, the goal of this analysis is only to illustrate that the observed offsets could plausibly be described by ram pressure, and that the inferred MW halo and disk gas densities needed to explain the magnitude of the ram pressure

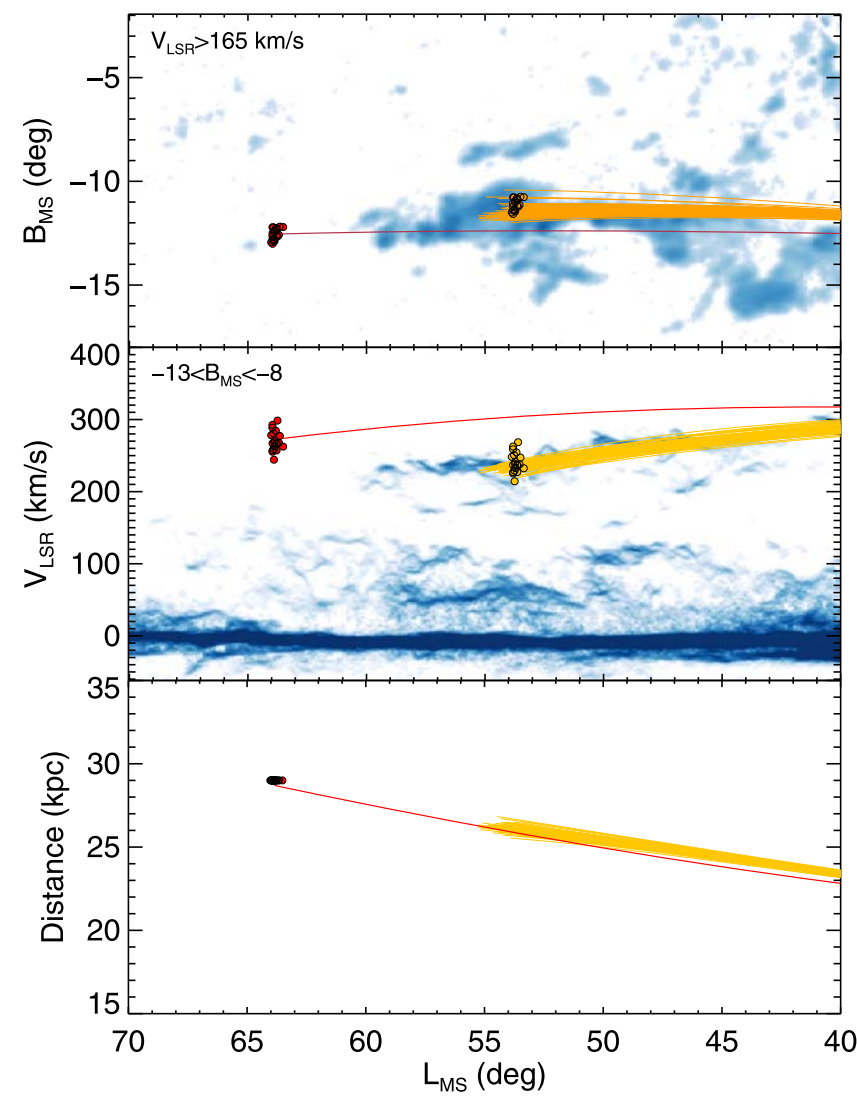

Figure 13. Orbits of both the mean PW 1 stars and the originating gas that experienced ram pressure. (Top) $B_{\mathrm{MS}}$ vs. $L_{\mathrm{MS}}$, (middle) $V_{\mathrm{LSR}}$ vs. $L_{\mathrm{MS}}$, and (bottom) distance vs. $L_{\mathrm{MS}}$. The red line is the PW 1 orbit with no ram pressure, while the orange lines show 64 orbits of the PW 1 birth cloud drawn from the MCMC posterior distributions of the parameters.

drag are reasonable. In doing this, we neglect more complex density evolution of the LA gas, assume that the LA II gas, at least around the PW 1 birthplace, acts like a cloud (rather than a dissolving and morphologically varying gas filament), and assume that no supernovae ( $\mathrm{SNe}$ ) have impacted the orbital energy of the gas. Still, this result motivates more detailed simulation of the interaction between the LA and the MW.

\section{Discussion}

The distance, RV, metallicity, and orbit suggest that not only is PW 1 associated with LA II, but that it is also associated with the MCs and MS. The association of PW 1 with LA II permits a more nuanced view of the LA than has previously been feasible. Not only does PW 1 provide a distance measurement to the LA, but it also constrains its chemical, orbital, and dynamical properties, such as how it is affected by ram pressure from the MW hot halo. At the same time, affiliating PW 1 with the LA gas also explains some of its properties, as discussed below.

\subsection{The Spatial Morphology of PW 1}

One of the mysteries of PW 1 is its unusual spatial shape and elongated distribution. However, the spatial correlation of the HI LA II gas and the PW 1 stars suggests a natural explanation for this. The PW 1 stars do not represent one single cluster that has disrupted but rather is likely the outcome 
of multiple star formation events associated with high-density H I clumps in LA II. This is a common feature observed in jellyfish galaxies experiencing ram pressure in galaxy clusters. Therefore, it might be more appropriate to call PW 1 a star formation "complex" or association rather than a star cluster in the traditional sense.

\subsection{Origin of the $L A$}

The mean metallicity of PW 1 of $\approx-1.23$ is similar to the measured metallicity of the LA $([\mathrm{O} / \mathrm{H}] \approx-1.16$; Fox et al. 2018), the Magellanic Bridge ( $\approx-1.0$; Lehner et al. 2008), and the trailing MS $(\approx-1.2$; Fox et al. 2013a). There is a large range in the measured metallicities of MW $\mathrm{HI}$ high-velocity clouds (Wakker 2001), and, therefore, the similarity of the metallicities in these distinct systems supports the notion that the LA, MB, and MS all share a common origin. These metallicities are also consistent with the metallicity of the SMC $\sim 2$ Gyr ago. Therefore, all of these gas structures associated with the MCs likely originated mainly from the SMC from the same tidal event about 2 Gyr ago.

Despite the similar metallicities among the gas structures, the origin of the MS and LA are still debated. This is mainly because (1) the observational data are far from complete-e.g., the metallicity measurements are limited to small number of sight lines, and (2) there are some observed features that cannot be easily explained by the sole SMC origin (e.g., Fox et al. $2013 b$ ). In one of the recent theoretical studies Pardy et al. (2018) argued that both the LMC and SMC contributed to create the LA and MS gas features. However, another recent MS simulation work by Tepper-García \& Bland-Hawthorn (2018) suggested that the LA gas does not originate in the MCs because the ram pressure from the MW hot halo gas would prevent the gas from reaching its present position. If PW 1 is indeed affiliated with LA II, as we suggest here, then there is now observational evidence that the impact of ram pressure is overestimated in Tepper-García \& Bland-Hawthorn (2018). The key discriminant is the assumed $\rho_{\mathrm{MW}}$ in Tepper-García \& Bland-Hawthorn (2018) versus what we infer from our scenario for PW 1.

\subsection{No Natal Gas Disruption?}

The conditions for triggering star formation in the LA is not well understood. Based on the fact that PW 1 is the only known stellar component to date that is likely associated with LA II, the BC of PW 1 must have satisfied very special star formation conditions in the LA while passing through the Galactic midplane. Aside from the unknown star formation conditions of PW 1, there is another mystery: how has the morphology of the PW 1 BC remained mostly intact? Our analysis in Section 4.3 shows that the present-day spatial distribution of the associated $\mathrm{HI}$ gas resembles that of the PW 1 stars across $2.5 \mathrm{kpc}$.

A gas cloud that forms a young star cluster is disrupted when the first SN occurs. The SN explosions effectively act to distort the original spatial correlations between the gas and stars by injecting radiative and mechanical energy into the birth gas. Similar spatial distributions of the stars and gas after the shift of $\left(-10^{\circ} .15,+1.44\right)$ in $\left(L_{\mathrm{MS}}, B_{\mathrm{MS}}\right)$ indicate that PW $1 \mathrm{BC}$ did not undergo significant gas removal and/or gas destruction period at all, or at least not at significant level. This might only be possible in the absence of stellar feedback in the PW 1 BC.
One way to avoid the impact of stellar feedback on the gas cloud is not to have SN events. To test the possibility that no SNe occurred in PW 1, we compute the expected number of SN explosions in PW 1-like star clusters. Based on the presentday mass, age, and metallicity of PW 1, PARSEC stellar evolutionary models suggest that the initial mass of PW 1 is $\sim 1800 M_{\odot}$. We then simulate a $1800 M_{\odot}$ star cluster 20,000 times assuming a Kroupa initial mass function and count the number of SN explosion events in each star cluster. If we assume that all stars more massive than $8 M_{\odot}$ explode as $\mathrm{SNe}$ II, then all of the simulated PW 1-like clusters produce at least 1 core-collapse $\mathrm{SN} \sim 3 \mathrm{Myr}$ (a typical lifetime of a $8 M_{\odot}$ star) after its birth. Thus, this scenario is unsuitable to explain the similar present-day spatial pattern between the PW 1 and its BC.

Another way to avoid the gas disruption by stellar feedback is to spatially decouple the $\mathrm{BC}$ and the newly formed stars before the first SN explosion. Over $3 \mathrm{Myr}$, the PW 1 stars were able to travel $\sim 14.3 \mathrm{pc}$ (corresponding to $\sim 50$ lyr) away from the $\mathrm{BC}$ based on the orbital calculation in Section 4.5. This spatial decoupling due to ram pressure might prevent the PW 1 BC from being significantly disrupted by stellar feedback. If the PW 1 stars and gas were indeed decoupled before the first SN explosion, our assumption about no effect of $\mathrm{SN}$ on the stellar motions (Section 4.5) can be naturally justified.

\section{Summary}

We have obtained high-resolution Magellan+MIKE spectra of 28 candidates of PW 1, a young stellar association in the region of the LA. Our sample allows us to draw some important conclusions about the properties and origin of both PW 1 and the LA:

1. PW 1 has a median metallicity of $[\mathrm{Fe} / \mathrm{H}]=-1.23$ with a small scatter of $0.06 \mathrm{dex}$ and an inferred velocity of $V_{\mathrm{LSR}}=273.4 \mathrm{~km} \mathrm{~s}^{-1}$ with a dispersion of $11.0 \mathrm{~km} \mathrm{~s}^{-1}$. The derived stellar parameters $\left(T_{\mathrm{eff}}, \log g,[\mathrm{Fe} / \mathrm{H}]\right)$ are consistent with the young, metal-poor isochrone (116 Myr and $[\mathrm{Fe} / \mathrm{H}]=-1.1)$ that was determined in Paper I using photometry for proper motion selected members.

2. There is a strong correlation between the spatial patterns of the PW 1 stars and the high-density H I clumps of LA II with an offset of $\left(-10^{\circ} .15,+1.55\right)$ in $\left(L_{\mathrm{MS}}, B_{\mathrm{MS}}\right)$ (Figure 10).

3. Due to the similarity of metallicity, velocity, spatial patterns, and the distance of PW 1, we find that PW 1 likely originated from the LA II complex of the MS.

4. The orbit and metallicity of PW 1 and LA II associate them with the MCs and MS, in contrast to some recent claims to the contrary.

5. Using an orbital analysis of the PW 1 stars and the LA II gas, taking into account ram pressure from an MW model, we constrain the halo gas density at the orbital pericenter of PW 1 to be $\boldsymbol{n}_{\text {halo }}(17 \mathrm{kpc})=2.7_{-2.0}^{+3.4} \times$ $10^{-3}$ atoms $\mathrm{cm}^{-3}$ and the disk gas density at the midplane at $20 \mathrm{kpc}$ to be $\boldsymbol{n}_{\text {disk }}(20 \mathrm{kpc}, 0)=6.0_{-2.0}^{+1.5} \times$ $10^{-2}$ atoms cm$~^{-3}$. We also predict that the current distance of the PW 1 BC in LA II is $27 \mathrm{kpc}$.

Future work will investigate the detailed chemical abundances of PW 1 and how it compares to the MCs. 
We thank Carlos Allende Prieto for sharing his Synspec IDL wrapper and utility software. We also thank Andy Casey for general help in running his Cannon software. We thank Michael Strauss for organizing and scheduling our Magellan time. A.P.W. thanks Stephanie Tonnesen and Elena D'Onghia for helpful conversations. D.B. and N.W.E. thank Jane Luu for valuable advice and assistance in using the Goodman spectrograph at the SOAR telescope. We thank Andrew Fox and Kat Barger for useful discussions on the origin of PW 1 and the metallicity variations in the LA. We thank the anonymous referee for useful comments that improved the manuscript. Support for this work was provided by NASA through Hubble Fellowship grant \#51386.01 awarded to R.L. B. by the Space Telescope Science Institute, which is operated by the Association of Universities for Research in Astronomy, Inc., for NASA, under contract NAS 5-26555. This work presents results from the European Space Agency (ESA) space mission Gaia. Gaia data are being processed by the Gaia Data Processing and Analysis Consortium (DPAC). Funding for the DPAC is provided by national institutions, in particular the institutions participating in the Gaia MultiLateral Agreement (MLA). The Gaia mission website is https://www.cosmos.esa. int/gaia. The Gaia archive website is https://archives.esac.esa. int/gaia. This paper includes data gathered with the $6.5 \mathrm{~m}$ Magellan Telescopes located at Las Campanas Observatory, Chile.

Based on observations obtained at the Southern Astrophysical Research (SOAR) telescope, which is a joint project of the Ministério da Ciência, Tecnologia, Inovações e Comunicações (MCTIC) do Brasil, the U.S. National Optical Astronomy Observatory (NOAO), the University of North Carolina at Chapel Hill (UNC), and Michigan State University (MSU).

Facilities: Magellan:Clay (MIKE), SOAR (Goodman).

Software: CarPy: MIKE (Kelson et al. 2000; Kelson 2003), Synspec (Hubeny \& Lanz 2017), IRAF (Tody 1986, 1993), The Cannon (Casey et al. 2012; Ness et al. 2018), Astropy (The Astropy Collaboration et al. 2018), ga la (Price-Whelan 2017), IPython (Pérez \& Granger 2007), matplotlib (Hunter 2007), numpy (Walt et al. 2011), scipy (Jones et al. 2001).

\section{ORCID iDs}

David L. Nidever (1) https://orcid.org/0000-0002-1793-3689 Adrian M. Price-Whelan (1) https://orcid.org/0000-00030872-7098

Yumi Choi (1) https://orcid.org/0000-0003-1680-1884

Rachael L. Beaton (10 https://orcid.org/0000-0002-1691-8217

Terese T. Hansen (1) https://orcid.org/0000-0001-6154-8983

David Aguado 자 https://orcid.org/0000-0001-5200-3973

Rana Ezzeddine (ㄴ) https://orcid.org/0000-0002-8504-8470

Semyeong Oh (1) https://orcid.org/0000-0001-7790-5308

\section{References}

Bernstein, R., Shectman, S. A., Gunnels, S. M., Mochnacki, S., \& Athey, A. E. 2003, Proc. SPIE, 4841, 1694

Besla, G., Kallivayalil, N., Hernquist, L., et al. 2007, ApJ, 668, 949

Besla, G., Kallivayalil, N., Hernquist, L., et al. 2012, MNRAS, 421, 2109

Bovy, J. 2015, ApJS, 216, 29

Brown, A. G. A., Vallenari, A., Prusti, T., de Bruijne, J. H. J., et al. 2018, A\&A, 616, A1

Brueck, M. T., \& Hawkins, M. R. S. 1983, A\&A, 124, 216

Brüns, C., Kerp, J., Staveley-Smith, L., et al. 2005, A\&A, 432, 45
Byrd, R. H., Lu, P., Nocedal, J., \& Zhu, C. 1995, SIAM J. Sci. Comput., 16,1190

Carpenter, B., Gelman, A., Hoffman, M., et al. 2017, Journal of Statistical Software, 76, 1

Casetti-Dinescu, D. I., Moni Bidin, C., Girard, T. M., et al. 2014, ApJL, 784, L37

Casey, A. R., Hogg, D. W., Ness, M., et al. 2016, arXiv:1603.03040

Casey, A. R., Keller, S. C., \& Da Costa, G. 2012, AJ, 143, 88

Choi, Y., Nidever, D. L., Olsen, K., et al. 2018a, ApJ, 866, 90

Choi, Y., Nidever, D. L., Olsen, K., et al. 2018b, ApJ, 869, 125

Clemens, J. C., Crain, J. A., \& Anderson, R. 2004, Proc. SPIE, 5492, 331

Connors, T. W., Kawata, D., \& Gibson, B. K. 2006, MNRAS, 371, 108

Delhaye, J. 1965, in Galactic Structure, ed. A. Blaauw \& M. Schmidt (Chicago, IL: Univ. Chicago Press), 61

Diaz, J. D., \& Bekki, K. 2012, ApJ, 750, 36

D'Onghia, E., \& Fox, A. J. 2016, ARA\&A, 54, 363

Dorman, B., Rood, R. T., \& O'Connell, R. W. 1993, ApJ, 419, 596

Dormand, J., \& Prince, P. 1980, JCoAM, 6, 19

Erkal, D., Belokurov, V., Laporte, C. F. P., et al. 2019, MNRAS, 487, 2685

For, B.-Q., Staveley-Smith, L., \& McClure-Griffiths, N. M. 2013, ApJ, 764, 74

Foreman-Mackey, D., Hogg, D. W., Lang, D., \& Goodman, J. 2013, PASP, 125,306

Fox, A. J., Barger, K. A., Wakker, B. P., et al. 2018, ApJ, 854, 142

Fox, A. J., Richter, P., Wakker, B. P., et al. 2013a, ApJ, 772, 110

Fox, A. J., Richter, P., Wakker, B. P., et al. 2013b, Msngr, 153, 28

Fox, A. J., Wakker, B. P., Smoker, J. V., et al. 2010, ApJ, 718, 1046

Gardiner, L. T., \& Noguchi, M. 1996, MNRAS, 278, 191

Goodman, J., \& Weare, J. 2010, Communications in Applied Mathematics and Computational Science, 5, 65

Gordon, M. A. 1976, MExP, 12, 277

Guhathakurta, P., \& Reitzel, D. B. 1998, in ASP Conf. Ser. 136, Galactic Halos, ed. D. Zaritsky (San Francisco, CA: ASP), 22

Harris, J., \& Zaritsky, D. 2004, AJ, 127, 1531

Harris, J., \& Zaritsky, D. 2009, AJ, 138, 1243

Helmi, A., van Leeuwen, F., McMillan, P., DPAC, et al. 2018, A\&A, 616, A12

Hernquist, L. 1990, ApJ, 356, 359

Homan, M. D., \& Gelman, A. 2014, J. Mach. Learn. Res., 15, 1593, http:// jmlr.org/papers/volume15/hoffman14a/hoffman14a.pdf

Hubeny, I., \& Lanz, T. 2011, Synspec: General Spectrum Synthesis Program, ascl: 1109.022

Hubeny, I., \& Lanz, T. 2017, arXiv:1706.01859

Hunter, J. D. 2007, CSE, 9, 90

Jones, E., Oliphant, T., Peterson, P., et al. 2001, SciPy: Open Source Scientific Tools for Python, v1.2.1., http://www.scipy.org/

Kalberla, P. M. W., \& Kerp, J. 2009, ARA\&A, 47, 27

Kallivayalil, N., van der Marel, R. P., Alcock, C., et al. 2006, ApJ, 638, 772

Kallivayalil, N., van der Marel, R. P., Besla, G., Anderson, J., \& Alcock, C. 2013, ApJ, 764, 161

Kelson, D. D. 2003, PASP, 115, 688

Kelson, D. D., Illingworth, G. D., van Dokkum, P. G., \& Franx, M. 2000, ApJ, 531,159

Kunkel, W. E., Irwin, M. J., \& Demers, S. 1997, A\&AS, 122, 463

Laporte, C. F. P., Gómez, F. A., Besla, G., Johnston, K. V., \& Garavito-Camargo, N. 2018, MNRAS, 473, 1218

Lehner, N., Howk, J. C., Keenan, F. P., \& Smoker, J. V. 2008, ApJ, 678, 219

Lu, L., Savage, B. D., Sembach, K. R., et al. 1998, AJ, 115, 162

Majewski, S. R., Nidever, D. L., Muñoz, R. R., et al. 2009, in IAU Symp. 256 , The Magellanic System: Stars, Gas, and Galaxies, ed. J. T. Van Loon \& J. M. Oliveira (Cambridge: Cambridge Univ. Press), 51

Mastropietro, C., Moore, B., Mayer, L., Wadsley, J., \& Stadel, J. 2005, MNRAS, 363, 509

Mathewson, D. S., Cleary, M. N., \& Murray, J. D. 1974, ApJ, 190, 291

McClure-Griffiths, N. M., Pisano, D. J., Calabretta, M. R., et al. 2009, ApJS, 181,398

McClure-Griffiths, N. M., Staveley-Smith, L., Lockman, F. J., et al. 2008, ApJL, 673, L143

Miller, M. J., \& Bregman, J. N. 2013, ApJ, 770, 118

Muñoz, R. R., Majewski, S. R., Zaggia, S., et al. 2006, ApJ, 649, 201

Ness, M., Hogg, D. W., Rix, H.-W., Ho, A. Y. Q., \& Zasowski, G. 2015, ApJ, 808,16

Ness, M., Rix, H., Hogg, D. W., et al. 2018, ApJ, 853, 198

Nidever, D. L., Hasselquist, S., Hayes, C. R., et al. 2019a, arXiv:1901.03448

Nidever, D. L., Majewski, S. R., \& Butler Burton, W. 2008, ApJ, 679, 432

Nidever, D. L., Majewski, S. R., Butler Burton, W., \& Nigra, L. 2010, ApJ, 723,1618

Nidever, D. L., Majewski, S. R., Muñoz, R. R., et al. 2011, ApJL, 733, L10 
Nidever, D. L., Olsen, K., Choi, Y., et al. 2019b, ApJ, 874, 118

Noël, N. E. D., Conn, B. C., Read, J. I., et al. 2015, MNRAS, 452, 4222

Oh, S., Price-Whelan, A. M., Hogg, D. W., Morton, T. D., \& Spergel, D. N. 2017, AJ, 153, 257

Olano, C. A. 2004, A\&A, 423, 895

Olsen, K. A. G., Zaritsky, D., Blum, R. D., Boyer, M. L., \& Gordon, K. D. 2011, ApJ, 737, 29

Pagel, B. E. J., \& Tautvaisiene, G. 1998, MNRAS, 299, 535

Pardy, S. A., D’Onghia, E., \& Fox, A. J. 2018, ApJ, 857, 101

Patel, E., Besla, G., \& Sohn, S. T. 2017, MNRAS, 464, 3825

Pérez, F., \& Granger, B. E. 2007, CSE, 9, 21

Philip, A. G. D. 1976a, BAAS, 8, 352

Philip, A. G. D. 1976b, BAAS, 8, 532

Price-Whelan, A. M. 2017, JOSS, 2, 388

Price-Whelan, A. M., Nidever, D. L., Choi, Y., et al. 2019, ApJ, 887, 19

Putman, M. E., Gibson, B. K., Staveley-Smith, L., et al. 1998, Natur, 394, 752

Putman, M. E., Peek, J. E. G., \& Joung, M. R. 2012, ARA\&A, 50, 491

Putman, M. E., Staveley-Smith, L., Freeman, K. C., Gibson, B. K., \& Barnes, D. G. 2003, ApJ, 586, 170

Recillas-Cruz, E. 1982, MNRAS, 201, 473
Richter, P., Fox, A. J., Wakker, B. P., et al. 2013, ApJ, 772, 111

Richter, P., Fox, A. J., Wakker, B. P., et al. 2018, ApJ, 865, 145

Russell, S. C., \& Dopita, M. A. 1992, ApJ, 384, 508

Schönrich, R., Binney, J., \& Dehnen, W. 2010, MNRAS, 403, 1829

Stanimirović, S., Hoffman, S., Heiles, C., et al. 2008, ApJ, 680, 276

Tepper-García, T., \& Bland-Hawthorn, J. 2018, MNRAS, 478, 5263

The Astropy Collaboration, Price-Whelan, A. M., Sipőcz, B. M., et al. 2018, AJ, 156,123

Tody, D. 1986, Proc. SPIE, 627, 733

Tody, D. 1993, in ASP Conf. Ser. 52, Astronomical Data Analysis Software and Systems II, ed. R. J. Hanisch, R. J. V. Brissenden, \& J. Barnes (San Francisco, CA: ASP), 173

Vollmer, B., Cayatte, V., Balkowski, C., \& Duschl, W. J. 2001, ApJ, 561, 708 Wakker, B. P. 2001, ApJS, 136, 463

Walt, S. v. d., Colbert, S. C., \& Varoquaux, G. 2011, CSE, 13, 22

Wannier, P., Wrixon, G. T., \& Wilson, R. W. 1972, A\&A, 18, 224

Weisz, D. R., Dolphin, A. E., Skillman, E. D., et al. 2013, MNRAS, 431, 364

Wong, T., Hughes, A., Ott, J., et al. 2011, ApJS, 197, 16

Zhang, L., Casetti-Dinescu, D. I., Moni Bidin, C., et al. 2019, ApJ, 871, 99

Zivick, P., Kallivayalil, N., van der Marel, R. P., et al. 2018, ApJ, 864, 55 